\title{
Adaptive condition-based maintenance decision framework for deteriorating systems operating under variable environment and uncertain condition monitoring
}

\author{
K.T. Huynh ${ }^{\mathrm{a}, *}$, A. Barros ${ }^{\mathrm{a}}$, C. Bérenguer ${ }^{\mathrm{b}}$ \\ ${ }^{a}$ Université de technologie de Troyes - Institut Charles Delaunay and STMR UMR CNRS 6279 - 12, rue Marie Curie, \\ CS42060, 10004 Troyes cedex - France \\ ${ }^{b}$ Gipsa-lab ; CNRS ; Grenoble Institute of Technology - 11, rue des Mathématiques - BP46 - 38402 Saint Martin \\ d'Hères cedex - France
}

\begin{abstract}
The present paper deals with the efficient use of different types of monitoring information in optimizing condition-based maintenance (CBM) decision-making for a deteriorating system operating under variable environment. The degradation phenomenon of system is the fatigue crack growth which is modeled by a physics-based stochastic process. The environment process is assumed to be modeled by a time-homogenous Markov chain with finite state space. We suppose that the environmental condition is observed perfectly, while the crack depth can be assessed imperfectly through a non-destructive ultrasonic technique. As such, two kinds of indirect information are available on the system at each inspection time: environmental covariate and diagnostic covariate. Based on this set of information, two CBM strategies adaptive to environmental condition are developed. In the first one, the adaptation scheme is time-based, while in the second, it is condition-based. These maintenance strategies are compared one to each other and to a classical non-adaptive one to point out the performances of each adaptation scheme and hence the appreciation of using different information sources in maintenance decision-making.
\end{abstract}

Keywords: Adaptive maintenance decision, diagnostic covariate, environmental covariate, fatigue crack growth, indirect condition monitoring, maintenance cost model, particle filter.

\footnotetext{
${ }^{*}$ Corresponding author

Email addresses: tuan.huynh@utt.fr (K.T. Huynh), anne.barros@utt.fr (A. Barros), christophe.berenguer@grenoble-inp.fr (C. Bérenguer)
} 


\section{Introduction}

With the development of engineering structures and systems, maintenance operations play an important role in efforts to improve the durability, reliability and maintainability of industrial systems. The dissemination and the expansion of instrumentation techniques and sensor technologies impulse the integration of diversified monitoring information in describing the system behavior and providing reliable condition-based maintenance decisions. Currently, we observe an increased interest in the use of information on covariates, i.e., variables that are correlated to, but do not define directly or completely the underlying system state. In practice, covariates could be categorized into two classes: environmental covariates and diagnostic covariates [1]. Environmental covariates, including environmental settings, stress, load, etc. may accelerate or decelerate the failure time of a system. Whereas, diagnostic covariates, such as the vibration level of fitted rotating machinery, the level of metal particle in oil analysis, the fatigue crack growth of steam turbine, the thickness of brake pad, etc., are often generated by the asset failure mechanism. Furthermore, these covariates are collected by different ways. Environmental covariates, in many practical situations, usually vary between a finite number of states during a system operating period. This kind of data, therefore, can be directly observable with reasonable accuracy at very low costs. On the contrary, diagnostic covariates, due to e.g. the difficulty of placing sensors in the harsh environmental conditions in which the system operates, are not always straightforward to defined directly, and one has to resort to indirect condition monitoring techniques. Potential methods of such techniques include vibration based monitoring, oil analysis, infrared thermography, acoustic emission analysis and motor current analysis [2]. In this manner, these diagnostic covariates are usually collected at high costs and they are always affected by noise and disturbance. Under this consideration, both type of covariates and nature of monitoring techniques have significant impacts on the system evolution, so a good reliability model should not ignore these aspects. However, in the literature, very few models take into full account all these aspects. For example, many studies include both environmental and diagnostic covariates in modeling the system degradation [3-6], but the quality of the measurement are ignored (i.e., all covariates are assumed perfectly determined). Whereas, some authors deal with the indirect measurement nature of diagnostic covariates [7-10], but they do not integrate the influences of environmental covariates 
in their models. In this framework, the present paper aims to build a degradation and measurement model in which all the aforementioned aspects are taken into account. Such a model is meant to be more realistic, and offers a good case study for discussion about the relevance of different kind of monitoring information in maintenance decision-making.

As a test case, we consider the well-known fatigue crack growth degradation phenomenon of a singleunit system operating under variable environment. The environmental covariate affects the speed and the variance of the crack growth. The crack depth is unobservable directly because the inspection method relies on classical non-destructive ultrasonic techniques [11]. Hence, modeling such a system leads to describe the characteristics of the operating environment and the system degradation evolution, the relation between environmental covariate, real degradation and diagnostic covariate, as well as the direct and indirect nature of monitoring techniques. This kind of model can be built by extending the degradation and measurement model studied in [9-12]. It is based on a Markovian stressful environment, a non-stationary Markovian process for the degradation phenomenon, a proportional hazard model to describe the impact of environment on the degradation, and a logit model for the indirect monitoring.

Based on the proposed degradation and measurement model, our aim is to develop a CBM framework in which the information on both diagnostic and environmental covariates is used efficiently. In CBM area, the periodic inspection and replacement strategy, denoted as $(\Delta T, M)$ strategy, is widely used because of its simple implementation [6]. With this kind of strategy, any intervention (i.e., inspection, replacement, etc.) is only possible at periodic prefixed times. However, this classical strategy seems inefficient to guarantee the system availability since at the same time it might "over inspect" or "under inspect" failure times [13]. This leads us to introduce adaptive maintenance strategies which allow avoiding more efficiently inopportune maintenance spending. In the literature, some adaptive strategies are proposed [14-17], but none of them has discussed about the time-based and conditionbased nature of adaptation scheme. The present paper, therefore, adds a new dimension to the adaptive strategies by proposing two separate maintenance decision-making approaches adaptive to the environmental condition. In the first one, the adaptation relies on the inspection periods, while in the second, it relies on the degradation-based preventive replacement thresholds of the system. The cost model of these strategies are developed, optimized and compared with the classical $(\Delta T, M)$ strategy. The com- 
parison results allow to assess the performance of each adaptation approach, and hence to make some conclusions on the interest of the using different information sources in maintenance decision-making.

The remainder of this paper is organized as follows. Section 2 of the paper is devoted to model the considered system. In Section 3, we present the adaptive CBM framework: the maintenance assumptions, the proposed strategies and the criterion to evaluate their performance. Section 4 deals with the numerical solution of the maintenance models considered in Section 3. In Section 5, we discuss the performance and the robustness of the adaptive CBM framework by investigating its sensitivities to different situations of intervention costs, environment characteristics and parameters estimation errors. Finally, we conclude the paper in Section 6 .

\section{System modeling and condition monitoring}

As a motivating exemple for the presented work, we are interested in the fatigue crack growth degradation phenomenon of a mechanical system that operates under a variable environment. The system fails when its crack depth exceeds a critical material thickness $d$. The crack depth is monitored indirectly by a non-destructive ultrasonic inspection technique, while the environmental state is observed directly. Such a system can be described by an extension of the degradation and measurement model considered in [9-12]. Compared to the original model, the one developed in this paper adds the impacts of environmental condition on the system degradation behavior and the different natures of condition monitoring technique.

\subsection{Degradation model without environmental impacts}

Degradation modeling of a mechanical equipment usually starts with a deterministic description of the underlying degradation phenomenon through physics or chemistry, then randomness can be incorporated into the model to preserve the stochastic nature of a degradation process [18]. In this work, the basis of the degradation model is the continuous time and deterministic Paris-Erdogan model which reflects the physical interpretation of the phenomenon

$$
\frac{d x}{d t}=C(\Delta K)^{n}
$$


where $x$ is the crack depth, $t$ is the time, $\Delta K=\Delta K(x)$ is the stress intensity amplitude, $C$ and $n$ are constant parameters related to the component material properties [19]. The parameters of the ParisErdogan model can be estimated from measured crack growth data [20]. The stress intensity factor is computed by $\Delta K=\beta \sqrt{x}$, where the parameter $\beta$ is related to the applied stress and geometrical configuration of the structure [21], and it is considered as constant when the impacts of operating environment are not taken into account [9-12].

To preserve the intrinsic randomness of the degradation phenomenon, the aforementioned deterministic Paris-Erdogan crack growth model is randomized. It has been suggested to randomize the deterministic crack propagation by a stochastic process [22] or by a randomization of characteristic parameters (i.e., $C, n$ or $\beta$ ) of the deterministic model [23]. The randomization approach used here is a special case of Yang's power law model [24] where a lognormal distributed random variable is introduced. The versatility of this randomization approach has been analyzed in [25] and justified on the basis of empirical data in $[26,27]$. Consequently, for a time step $\Delta t$ sufficiently small, one can discretize and insert the randomness into the equation (2.1) as follows [11]

$$
x_{t_{i}}=x_{t_{i-1}}+\mathrm{e}^{\omega_{t_{i}}} C\left(\beta \sqrt{x_{t_{i-1}}}\right)^{n} \Delta t
$$

where $\omega_{t_{i}} \sim \mathcal{N}\left(0, \sigma_{\omega}^{2}\right), i=1,2, \ldots$ are Gaussian random disturbances, $x_{t_{i}}$ is the crack depth at time step $t_{i}=i \Delta t$ with $0<x_{t_{i-1}}<x_{t_{i}}<+\infty$.

\subsection{Environmental modeling and degradation model with environmental impacts}

The aforementioned degradation model does not consider the influences of operating environment on the system behavior. This section, therefore, aims to incorporate this aspect into the above degradation model. In a way similar to the works in $[3,5,28]$, we model the evolution of stressful environment according to a time-homogeneous Markov chain with a discrete and finite state space. This choice is justified by the fact that the environmental impact on the system evolution is continuous and its repercussions are significant when the environmental states are really different. Let us denote $\Xi=\{1,2, \ldots, m\}$ the state space of the operating environment, and $e_{t_{i}}$ its state (i.e., environmental 
covariate) at time $t_{i}$. The evolution of $e_{t_{i}}$ in the state space $\Xi$ is expressed by the transition matrix

$$
\mathcal{T}_{\alpha}=\left(\tau_{k h}\right)_{1 \leq k \leq m, 1 \leq h \leq m}
$$

where $\tau_{k h}=P\left(e_{t_{i}}=h \mid e_{t_{i-1}}=k\right), \forall i=1,2, \ldots$, is the transition probability of the Markov chain $\left\{e_{t_{i}}\right\}_{t_{i} \geq 0}$ from the state $k$ at time $t_{i-1}$ to the state $h$ at time $t_{i}$. The parameter $\alpha \in[0,1]$ defines the transition speed between the states of $\left\{e_{t_{i}}\right\}_{t_{i} \geq 0}$.

When operating under variable environment, the speed and variance of the system degradation can be accelerated or decelerated according to the environmental covariate. In order to take into account this phenomenon, the characteristic parameters of the degradation model (2.2) are linked to the environmental covariate $e_{t_{i}}$. The parameters $C$ and $n$ only depend on the component material properties, hence do not depend on $e_{t_{i}}$. Since $\beta$ is the parameter related to the applied stress on the component, it is considered as a function $\beta\left(e_{t_{i}}\right)$ of environmental covariate $e_{t_{i}}$. To characterize concretely this relation, a model similar to the proportional hazards model proposed by Cox [29] is used.

$$
\beta\left(e_{t_{i}}\right)=\beta_{b} \cdot \exp \left(\gamma_{1} 1_{\left\{e_{t_{i}}=1\right\}}+\gamma_{2} 1_{\left\{e_{t_{i}}=2\right\}}+\cdots+\gamma_{m} 1_{\left\{e_{t_{i}}=m\right\}}\right)=\beta_{b} \cdot \mathrm{e}^{\gamma_{t_{t_{i}}}},
$$

where $\beta_{b}$ is the constant baseline parameter related to the normal environment (i.e., there is no additional stress due to the severe condition of operating environment), $\gamma_{k} \geq 0, k=1,2, \ldots, m$, is the regression parameters that reflect additional impact of the stressful environment on the system degradation behavior $\left(\gamma_{e_{t_{i}}} \in\left\{\gamma_{1}, \gamma_{2}, \ldots, \gamma_{m}\right\}\right)$, and $1_{\{\cdot\}}$ denotes the indicator function which equals 1 if the argument is true and 0 otherwise. Consequently, from equation (2.2), the degradation model with the impact of operating environment can be expressed as

$$
x_{t_{i}}=x_{t_{i-1}}+\mathrm{e}^{\omega_{t_{i}}} C\left(\beta\left(e_{t_{i}}\right) \cdot \sqrt{x_{t_{i-1}}}\right)^{n} \Delta t,
$$

where the function $\beta\left(e_{t_{i}}\right)$ is given by the equation (2.4). This model allows the recursive calculation of the crack depth $x_{t_{i}}$ at each time step $t_{i}$ given the the model parameters, the crack depth $x_{t_{i-1}}$ at time step $t_{i-1}$, and the environmental covariate $e_{t_{i}}$ at time step $t_{i}$.

The fundamental differences between the new degradation model and the original one come from 
the environment aspect, it is then interesting to study the influences of environment characteristics on the degradation behavior. Understanding thoroughly these influences helps us propose appropriate maintenance strategies for the considered system. We consider firstly the stress level of environment. From equation (2.5), we can easily show that the system degradation rate $\Delta x_{t_{i}}=\frac{x_{t_{i}}-x_{t_{i-1}}}{\Delta t}$ follows a lognormal distribution

$$
\Delta x_{t_{i}} \sim \log \mathcal{N}\left(\ln \left(C\left(\beta\left(e_{t_{i}}\right) \sqrt{x_{t_{i-1}}}\right)^{n}\right), \sigma_{\omega}^{2}\right)
$$

Thus, the mean and variance of $\Delta x_{t_{i}}$ are given respectively by

$$
E\left[\Delta x_{t_{i}}\right]=C\left(\beta\left(e_{t_{i}}\right) \sqrt{x_{t_{i-1}}}\right)^{n} \mathrm{e}^{\frac{\sigma_{\omega}^{2}}{2}} \quad \text { and } \quad \operatorname{var}\left[\Delta x_{t_{i}}\right]=E^{2}\left[\Delta x_{t_{i}}\right] \cdot\left(\mathrm{e}^{\sigma_{\omega}^{2}}-1\right) .
$$

As these quantities are increasing functions of $\beta\left(e_{t_{i}}\right)$, the higher the stress level of environment, the more the system lifetime is shortened and variable. Consider now the influences of the transition speed between environmental states (i.e., parameter $\alpha$ ) illustrated through a numerical exemple. A single-unit system operating under a 2-states Markovian environment is considered. The parameters of the model are chosen as $C=0.015, n=0.35, b_{b}=3.9, \sigma_{\omega}^{2}=2.53, \Delta t=0.2$. For the 2-state Markovian environment (i.e., $\left.\Xi=\{1,2\}, e_{t_{0}}=1\right), e_{t_{i}}=1$ and $e_{t_{i}}=2$ correspond respectively to the normal condition $\left(\gamma_{1}=0\right)$ and the stressful condition $\left(\gamma_{2}=6\right)$, and the transition matrix is chosen by

$$
\mathcal{T}_{\alpha}=\left(\begin{array}{cc}
1-\alpha & \alpha \\
\alpha & 1-\alpha
\end{array}\right)
$$

According to this transition matrix, the higher the value of $\alpha$, the faster the transition of environment [5]. The critical material thickness (i.e., failure threshold) is chosen as $d=9$. The numerical results for the two cases $\alpha=0.003$ and $\alpha=0.12$ are shown in Figure 1. Sub-figures 1a and 1b illustrate the evolution of the system degradation under the different situations of the transition speed of operating environment. From Sub-figures 1c and 1d, we remark that the degradation process and the failure times are more scattered when the transition speed is lower. This phenomenon is comprehensible when considering the percentage of time intervals related to the stressful environment in a life cycle of the system. When the environmental state varies slowly, some life cycles can be totally non-stressed, 

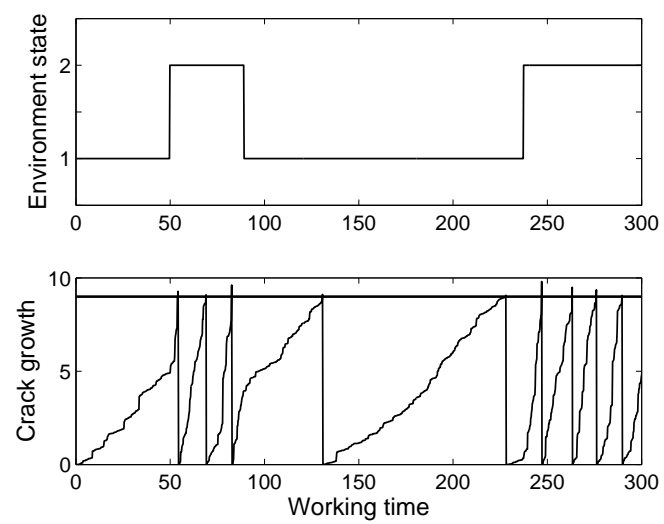

(a) System evolution - $\alpha=0.003$
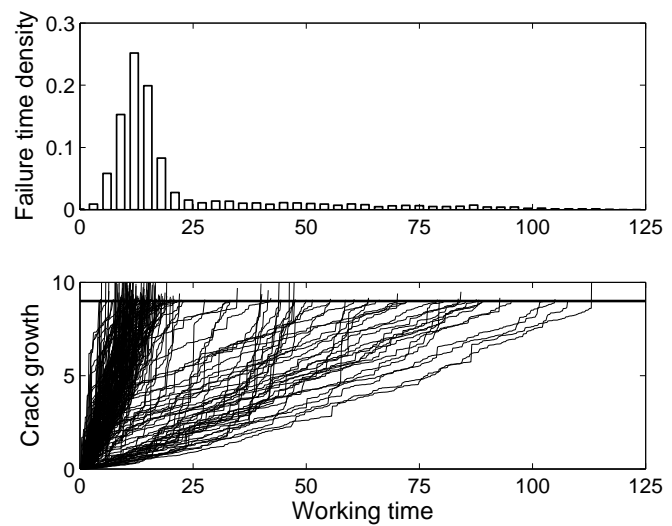

(c) Characteristic of degradation process - $\alpha=0.003$
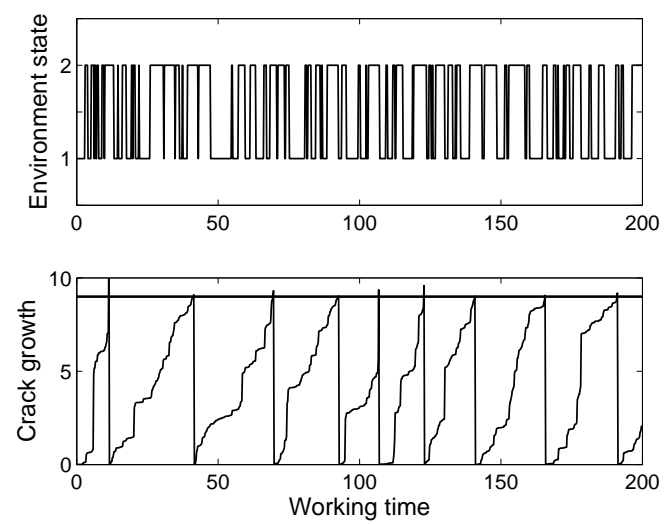

(b) System evolution $-\alpha=0.12$
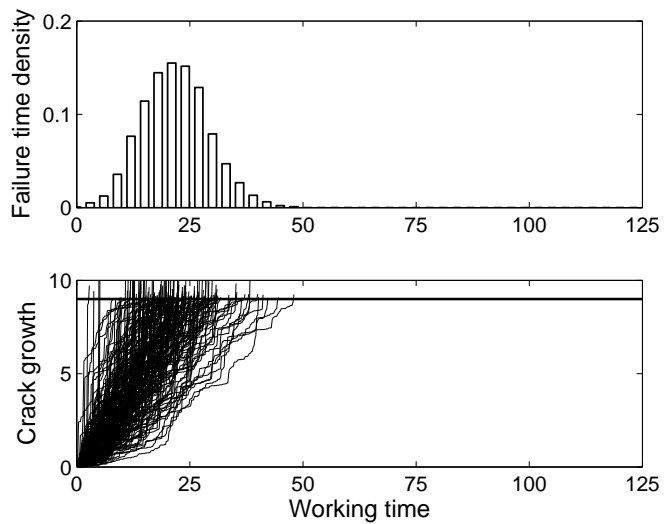

(d) Characteristic of degradation process $-\alpha=0.12$

Figure 1: Influence of transition speed of operating environment on the system degradation

whereas some others can be completely stressed (see Sub-figure 1a). On the contrary, when the environmental state changes quickly, all the life cycles become more or less similar (see Sub-figure 1b). Since the system degradation behavior is driven by these percentages, the variance of the failure time variance is larger for the lower transition speed of environment.

\subsection{Condition monitoring models}

Condition monitoring provides useful information to assess the system reliability and to make a maintenance decision. For the considered system, the environmental state and the crack depth are under monitoring. The condition of operating environment, in many practical situations, are easy to 
identify. This is particularly true in the case of monitoring the temperature and humidity conditions, or the rate of a production line. This is why we suppose that the environmental state $e_{t_{i}}$ at time $t_{i}$ is directly observed without errors and with a negligible cost. Contrary to the environmental state, the crack depth $x_{t_{i}}$ at time step $t_{i}$ is not directly observable, but can be assessed through a classical non-destructive ultrasonic technique with a non-negligible inspection cost. In the same way as in [30], a logit model is used to describe this inspection technique. As such, instead of the true crack depth $x_{t_{i}}$, the inspection returns the noisy measurement $z_{t_{i}}$ that correlates with $x_{t_{i}}$ through the expression $[11]$

$$
\ln \frac{z_{t_{i}}}{d-z_{t_{i}}}=\beta_{0}+\beta_{1} \ln \frac{x_{t_{i}}}{d-x_{t_{i}}}+v_{t_{i}}
$$

where $d$ is the critical material thickness of component, $\beta_{0} \in \mathbb{R}$ and $\beta_{1}>0$ are parameters to be estimated from experimental data, and $v_{t_{i}}$ is a white Gaussian noises such that $v_{t_{i}} \sim \mathcal{N}\left(0, \sigma_{v}^{2}\right)$. From the mathematical aspect, Equation (2.9) is valid for all $x_{t_{i}} \in(0, d)$. The measurement variable $z_{t_{i}}$ is thereby a random variable which is defined in the domain $0<z_{t_{i}}<d$ for all $x_{t_{i}} \in(0, d)$. From Equation (2.9), given $x_{t_{i}} \in(0, d)$, the likelihood probability density function (pdf) of the measurement $z_{t_{i}}$ is derived as [9]

$$
f\left(z_{t_{i}} \mid x_{t_{i}}\right)=\frac{1}{\sqrt{2 \pi \sigma_{v}^{2}}} \mathrm{e}^{-\frac{1}{2 \sigma_{v}^{2}}\left(\ln \frac{z_{t_{i}}}{d-z_{t_{i}}}-\beta_{0}-\beta_{1} \ln \frac{x_{t_{i}}}{d-x_{t_{i}}}\right)^{2}} \frac{d}{z_{t_{i}}\left(d-z_{t_{i}}\right)} \cdot 1_{\left\{0<z_{t_{i}}<d\right\}} .
$$

Consequently, the system condition monitoring returns two types of information at each inspection time: environmental covariate $e_{t_{i}}$ and diagnostic covariate $z_{t_{i}}$. In the next section, we show how to use these covariates to make an adaptive maintenance decision.

\section{Adaptive condition-based maintenance framework}

We introduce in this section two CBM strategies adaptive to the environmental covariate. A so called $\left(\Delta T_{k}, M\right)$ strategy represents the time-based adaptation approach and a so called $\left(\Delta T, M_{k}\right)$ strategy represents the condition-based adaptation approach. Since the diagnostic covariates cannot reflect precisely the degradation state of system, the decision rules of both strategies rely on the estimated degradation level reconstructed from these noisy covariates. 


\subsection{Assumptions and objective cost function}

We suppose that the system degradation (i.e., the crack depth) is hidden and the system failure is non-self-announcing. This means that the system reveals only its degradation state and its failure through a monitoring procedure. Continuous monitoring performed at each time step $\Delta t$ is usually very costly and sometimes impossible in practical engineering applications. In this framework, it is more suitable to implement discrete monitoring with an inter-inspection length taken as a multiple of $\Delta t$. An indirect non-destructive ultrasonic technique is then used to inspect the system, so the noisy measurement (i.e., diagnostic covariate) $z_{T_{i}}$ and the working/failure state of the system are collected at each discrete inspection time $T_{i}$ with a cost $C_{i}$. These assumptions are reasonable because in practice, for economic and safety reasons, the system is considered as "failed" as soon as a defect or an important deterioration appears, even if it is still running, so no indicator can exhibit the degradation and failure state of system except to do an inspection. The condition of stressful environment (i.e., environmental covariate) is assumed to be perfectly collected with a negligible cost because it is much more easy to detect compared to the system degradation state. Also, we assume that the inspection takes negligible time.

Two maintenance operations are available on the system: the preventive replacement with cost $C_{p}>C_{i}$ and the corrective replacement with cost $C_{c}$. A replacement can be either a true physical replacement or an overhaul or repair such that the system is as-good-as-new after the repair. Even though both the preventive and the corrective maintenance actions put the system back in the asgood-as-new state, they are not necessarily identical in practice because the corrective replacement (or renewal) is unplanned and it has to be performed on a more deteriorated system, and the cost $C_{c}$ can also comprise different costs associated with failure. It is thus likely to be more complex and more expensive (i.e., $C_{c}>C_{p}$ ). Moreover a replacement, whether preventive or corrective, can only be instantaneously performed at predetermined inspection times. Therefore, there exists a system inactivity after failure and an additional cost is incurred from the failure time until the next replacement time at a cost rate $C_{d}$.

To assess the performance of a maintenance strategy, one usually uses cost-based criteria in the literature. The maintenance cost of a maintenance strategy can be computed over a bounded or unbounded time horizon [31]. The unbounded time horizon model seems to be more advantage in 
making repeated investment decisions, because it does not ignore the future [32]. Thus, in the aim of long-term exploitation of a system, we focus on the widely used expected maintenance cost per unit over an infinite time span to assess the performance of the proposed strategies. The assumption of an as-good-as-new maintained system leads to the use of the regenerative properties of system state to compute the cost rate [33]

$$
C_{\infty}=\lim _{t \rightarrow \infty} \frac{C(t)}{t}=\frac{E[C(S)]}{E[S]}
$$

where $C(t)$ is the cumulated maintenance cost at time $t, S$ is the time between two successive renewals.

\subsection{Motivations for an adaptive decision to environmental covariate}

We are interested in the construction of dynamic CBM strategies adaptive to environmental covariate. The main idea is to begin with the periodic inspection and replacement $(\Delta T, M)$ strategy proposed by Huynh et al. in [6]. As mentioned in Section 1, according to this maintenance policy, any intervention (i.e. inspection, replacement, etc.) is only possible at periodic prefixed times $T_{i}=i \cdot \Delta T$, $i=1,2, \ldots$. At each inspection, if the estimated degradation level reconstructed from diagnostic covariates exceeds a threshold $M$ and no failure occurred, a preventive replacement is carried out. But upon inspection, if the system is detected to be in failure state, it is correctively replaced. The periodic inter-inspection interval $\Delta T$ and the preventive replacement threshold $M$ are the two decision variables of this maintenance strategy (i.e., we seek the optimal values $\left(\Delta T_{o p t}, M_{\text {opt }}\right)$ which minimize the cost criterion (3.1)). As such, decision rules of the $(\Delta T, M)$ strategy are indirectly relied on the actual value of diagnostic covariates and the average behavior of environmental covariate. In fact, this strategy is quite efficient especially when compared with a time-based maintenance strategy [6,34]. However, it is not yet an adequate maintenance policy strategy in some situations, because it does not take into account the current state of environmental covariate which can also bring out much significant information on the health state of system and its future life. So it can be interesting to propose maintenance strategies which can incorporate both actual diagnostic and environmental covariates in maintenance decision-making.

As shown in Section 2, for the considered system, the speed and the variance of crack growth are increasing functions of the stress level of environment. So the maintenance decisions should be adaptive to environmental covariate. From the $(\Delta T, M)$ strategy, a natural approach is to adapt one 
or both decision parameters $\Delta T$ and $M$ to the environmental covariate. For example, the intervention actions should be carried out more frequently (by e.g., shortening the inter-inspection interval) and/or the safety zone of system should be extended (by e.g., lowering the preventive replacement threshold) for a stressful condition rather than for a non-stressful condition. As such, one can have more precise information on the actual condition of system and its future evolution, hence can avoid more efficiently the system failures. Thus, the optimal values of the parameters $\Delta T$ and/or $M$ are no longer fixed for all the system lifetime as in $(\Delta T, M)$ strategy, but vary with the environmental covariate. In the following, two adaptive CBM strategies called $\left(\Delta T_{k}, M\right)$ and $\left(\Delta T, M_{k}\right)$ are developed. The $\left(\Delta T_{k}, M\right)$ strategy where inter-inspection intervals vary according to the current environmental state represents a time-based adaptation approach, while the $\left(\Delta T, M_{k}\right)$ strategy where preventive replacement thresholds vary according to the current environmental state represents a condition-based adaptation approach. Of course, a strategy where these both parameters adaptive to the environmental condition may be interesting and be a general policy of both $\left(\Delta T_{k}, M\right)$ strategy and $\left(\Delta T, M_{k}\right)$ strategy. However, such a strategy seems redundant to the paper scope and we content ourselves with considering separately one of the two adaptation approaches at each time.

\subsection{Adaptive condition-based maintenance decision with multi-periods of inspection}

In order to highlight the time-based adaptation approach to environmental covariate, we propose here a $\left(\Delta T_{k}, M\right)$ strategy that represents an adaptive CBM decision structure with multi-periods of inspection. Such a strategy is similar to the $(\Delta T, M)$ strategy, except that its inspection periods can adapt to different states of the stressful environment. However, for the same stress level, e.g., $e_{t_{i}}=k$, $k \in\{1,2, \ldots, m\}$, these periods remain constant and are equal to $\Delta T_{k}$.

\subsection{1. $\left(\Delta T_{k}, M\right)$ strategy}

The decision structure of the $\left(\Delta T_{k}, M\right)$ strategy in a renewal cycle can be described by the following scheme. The system is regularly inspected with a cost $C_{i}$ and with a period $\Delta T_{k}$ according to the state $e_{t_{i}}=k$ of environment. The inspection returns the actual stressful level of operating environment, the noisy measurement of the system degradation, as well as its working/failure state. At each inspection time $T_{i}, i=1,2, \ldots$, if the system fails (i.e., $x_{T_{i}} \geq d$ ), it is correctively replaced with a cost $C_{c}$, and its 
downtime generates an additional cost at a rate $C_{d}$. But if the system is still working (i.e., $x_{T_{i}}<d$ ), given the environmental covariate $e_{T_{i}}=k$ and the sequence of diagnostic covariates up to time $T_{i}$, $\mathbf{Z}_{T_{0: i}}=\left\{z_{T_{0}}, z_{T_{1}}, \ldots, z_{T_{i}}\right\}$, the estimated degradation level $\widehat{x}_{T_{i}}$ of the system is constructed. We make then a maintenance decision conditional on $\widehat{x}_{T_{i}}$ as follows

- If $\widehat{x}_{T_{i}} \geq M$, a preventive replacement is done with a $\operatorname{cost} C_{p}$.

- If $\widehat{x}_{T_{i}}<M$, nothing is done and the decision is postponed until the next inspection time $T_{i+1}$ that depends on the stress level $e_{T_{i}}$ observed at $T_{i}$ (e.g., $T_{i+1}=T_{i}+\Delta T_{k}$ if $e_{T_{i}}=k$ ).

Under this adaptive maintenance policy, the preventive replacement threshold $M$ and the inspection periods $\Delta T_{1}, \ldots, \Delta T_{m}$ are the decision variables. Figure 2 shows a schematic evolution of the maintained system state under the $\left(\Delta T_{k}, M\right)$ strategy when the state space of the operating environment is chosen by $\Xi=\{1,2\}$. We remark that the $\left(\Delta T_{k}, M\right)$ strategy returns to the $(\Delta T, M)$ strategy when

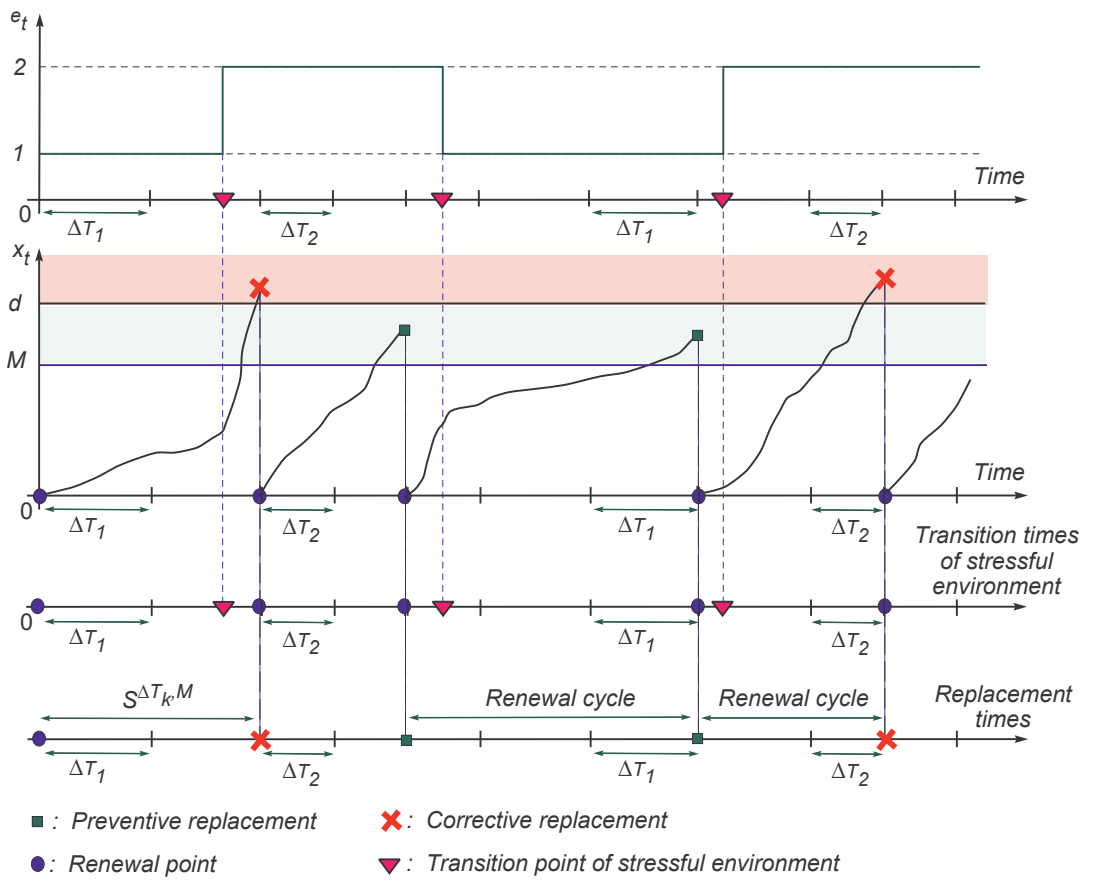

Figure 2: Illustration of the decision rule of $\left(\Delta T_{k}, M\right)$ strategy

the inspection periods $\Delta T_{k}, k=1,2, \ldots, m$ are equal, the former is more general and then more profitable than the latter. This reflects the benefit of the time-based adaptation approach to environmental 
covariate.

\subsubsection{Maintenance cost model}

To assess the performance of the $\left(\Delta T_{k}, M\right)$ strategy, one relies on its mathematical cost model. Applying Equation (3.1), the long-run expected maintenance cost rate of the $\left(\Delta T_{k}, M\right)$ strategy is computed by

$$
C_{\infty}^{\Delta T_{k}, M}\left(\Delta T_{1}, \ldots, \Delta T_{m}, M\right)=\frac{C_{i} E\left[N_{i}^{\Delta T_{k}, M}\right]+C_{p} P_{p}^{\Delta T_{k}, M}+C_{c}\left(1-P_{p}^{\Delta T_{k}, M}\right)+C_{d} E\left[W_{d}^{\Delta T_{k}, M}\right]}{E\left[S^{\Delta T_{k}, M}\right]}
$$

where $P_{p}^{\Delta T_{k}, M}, N_{i}^{\Delta T_{k}, M}$ and $W_{d}^{\Delta T_{k}, M}$ are respectively the preventive replacement probability, the number of inspections, and the system inactivity interval in a replacement cycle $S^{\Delta T_{k}, M}$ under the $\left(\Delta T_{k}, M\right)$ strategy. Thus, the optimization of this strategy is reduced to find the optimal values of decision variables $\left(\Delta T_{1, o p t}, \ldots, \Delta T_{m, o p t}, M_{o p t}\right)$ which minimize the cost rate $C_{\infty}^{\Delta T_{k}, M}$

$$
C_{\infty}^{\Delta T_{k}, M}\left(\Delta T_{1, o p t}, \ldots, \Delta T_{m, o p t}, M_{o p t}\right)=\min _{\Delta T_{1}, \ldots, \Delta T_{m}, M}\left\{C_{\infty}^{\Delta T_{k}, M}\left(\Delta T_{1}, \ldots, \Delta T_{m}, M\right)\right\}
$$

The optimal solution of the $\left(\Delta T_{k}, M\right)$ strategy is obtained if we know the mathematical formulas of $P_{p}^{\Delta T_{k}, M}, E\left[N_{i}^{\Delta T_{k}, M}\right], E\left[W_{d}^{\Delta T_{k}, M}\right]$ and $E\left[S^{\Delta T_{k}, M}\right]$. However, as in [10], we can show that the analytical formulas of these quantities are almost impossible to derive due to the complexity of the proposed degradation and measurement model, and the adaptive maintenance decision rule based on estimated degradation state and environmental covariate. In Section 4, we use numerical approaches (i.e., particle filter technique for state estimation, and Monte Carlo simulation for maintenance optimization) to overcome the problem.

\subsection{Adaptive condition-based maintenance decision with multi-thresholds for preventive replacement}

To highlight the condition-based adaptation approach to environmental covariate, we propose in this subsection a maintenance strategy with multi-thresholds for preventive replacement (i.e., $\left(\Delta T, M_{k}\right)$ strategy). For such a strategy, the inspection period $\Delta T$ is always constant regardless of environmental condition, while the preventive replacement thresholds $M_{k}, k \in\{1,2, \ldots, m\}$ can vary depending on 
the stress level of environment. However, under the same stress level, e.g., $e_{t_{i}}=k$, the threshold remains constant is equal to $M_{k}$.

\subsection{1. $\left(\Delta T, M_{k}\right)$ strategy}

The decision structure of the $\left(\Delta T, M_{k}\right)$ strategy in a renewal cycle can be described by the following scheme. The system is periodically inspected with a cost $C_{i}$ and with a period $\Delta T$ regardless of the environmental condition. The inspection returns the actual stressful level of operating environment, the noisy measurement of the system degradation, as well as its working/failure state. At each inspection time $T_{i}=i \Delta T, i=1,2, \ldots$, if the system fails (i.e., $x_{T_{i}} \geq d$ ), it is correctively replaced a cost $C_{c}$, and its downtime generate an additional cost at a rate $C_{d}$. But if the system is still working (i.e., $x_{T_{i}}<d$ ), given the environmental covariate $e_{T_{i}}=k$ and the sequence of diagnostic covariates up to time $T_{i}$, $\mathbf{Z}_{T_{0: i}}=\left\{z_{T_{0}}, z_{T_{1}}, \ldots, z_{T_{i}}\right\}$, the estimated degradation level $\widehat{x}_{T_{i}}$ of the system is constructed. We make then a maintenance decision conditional on $\widehat{x}_{T_{i}}$ as follows

- If $e_{T_{i}}=k$ and $\widehat{x}_{T_{i}} \geq M_{k}$, a preventive replacement is done with a cost $C_{p}$.

- If $e_{T_{i}}=k$ and $\widehat{x}_{T_{i}}<M$, nothing is done and the decision is postponed until the next inspection time $T_{i+1}=(i+1) \Delta T$.

Under this adaptive maintenance policy, the preventive replacement thresholds $M_{1}, \ldots, M_{m}$ and the inspection period $\Delta T$ are the decision variables. Figure 3 shows a schematic evolution of the maintained system state under the $\left(\Delta T_{k}, M\right)$ strategy when he state space of the operating environment is chosen by $\Xi=\{1,2\}$. It is noticed that the $\left(\Delta T, M_{k}\right)$ strategy returns to the $(\Delta T, M)$ strategy when the inspection periods $M_{k}, k=1,2, \ldots, m$ are equal, the former is more general and then more profitable than the latter. This reflects the benefit of the condition-based adaptation approach to environmental covariate.

\subsubsection{Maintenance cost model}

To assess the performance of the $\left(\Delta T, M_{k}\right)$ strategy, one relies on its mathematical cost model. Applying Equation (3.1), the long-run expected maintenance cost rate of the $\left(\Delta T, M_{k}\right)$ strategy is 


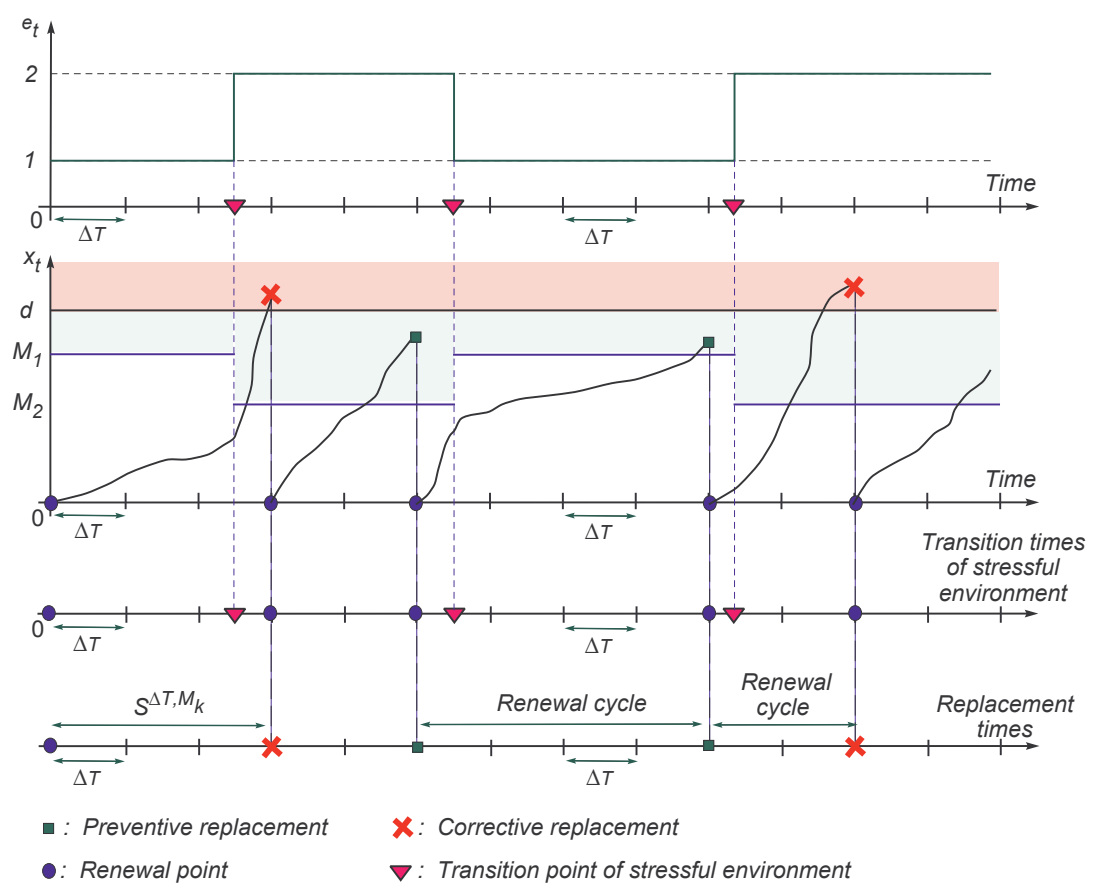

Figure 3: Illustration of the decision rule of $\left(\Delta T, M_{k}\right)$ strategy

expressed by

$$
C_{\infty}^{\Delta T, M_{k}}\left(\Delta T, M_{1}, \ldots, M_{m}\right)=\frac{C_{i} E\left[N_{i}^{\Delta T, M_{k}}\right]+C_{p} P_{p}^{\Delta T, M_{k}}+C_{c}\left(1-P_{p}^{\Delta T, M_{k}}\right)+C_{d} E\left[W_{d}^{\Delta T, M_{k}}\right]}{E\left[S^{\Delta T, M_{k}}\right]}
$$

where $P_{p}^{\Delta T, M_{k}}, N_{i}^{\Delta T, M_{k}}$ and $W_{d}^{\Delta T, M_{k}}$ are respectively the preventive replacement probability, the number of inspections, and the system inactivity interval in a replacement cycle $S^{\Delta T, M_{k}}$ under the $\left(\Delta T, M_{k}\right)$ strategy. Thus, the optimization of this strategy is reduced to find the optimal values of decision variables $\left(\Delta T_{o p t}, M_{1, \text { opt }}, \ldots, M_{m, o p t}\right)$ which minimize the cost rate $C_{\infty}^{\Delta T, M_{k}}$

$$
C_{\infty}^{\Delta T, M_{k}}\left(\Delta T_{o p t}, M_{1, o p t} \ldots, M_{m, o p t}\right)=\min _{\Delta T, M_{1} \ldots, M_{m}}\left\{C_{\infty}^{\Delta T, M_{k}}\left(\Delta T, M_{1} \ldots, M_{m}\right)\right\}
$$

The optimal solution of the $\left(\Delta T, M_{k}\right)$ strategy is obtained if we know the mathematical formulas of $P_{p}^{\Delta T, M_{k}}, E\left[N_{i}^{\Delta T, M_{k}}\right], E\left[W_{d}^{\Delta T, M_{k}}\right]$ and $E\left[S^{\Delta T, M_{k}}\right]$. However, in the same reason as in the above subsection, the analytical formulas of these quantities are almost impossible to derive, and we shall 
use the particle filter technique and the Monte Carlo simulation to overcome this problem.

\section{Numerical solution for adaptive condition-based maintenance models}

As shown in Section 3, the analytical analysis of the proposed maintenance strategies is not trivial due to the complexity of the degradation and measurement model, and the adaptive decision rules based on both estimated degradation state and environmental covariate. This is why we propose in this section numerical methods to overcome this difficulty. The particle filter approach is used for the online state estimation, while Monte Carlo simulations procedure is used to assess the performance of maintenance strategies.

\subsection{Particle filtering state estimation}

The measurements given from the ultrasonic inspection technique are a useful information for maintenance decision-making. However, a direct use of such information does not provide reliable results due to the presence of measurement uncertainty. Estimating the true state of system degradation from these noisy measurements is thereby necessary. In the literature, stochastic filtering [35], proportional hazards modeling [36] and hidden Markov models [37] are the three common data-driven approaches for inferring the underlying system state from noisy monitoring data. The proportional hazards model is restricted to its proportional assumption, and the hidden Markov model is applied only for discrete state variables. The stochastic filtering can overcome these drawbacks. Moreover, because the stochastic filtering uses the full history of condition monitoring data, it outperforms the others [38]. The performance of stochastic filtering is limited with high dimensional data, however this is not the problem in our case study because the degradation phenomenon is a single fatigue crack growth path.

Particle filtering is a numerical version of stochastic filtering. It is a Monte Carlo-based computation tool particularly useful for optimal estimation and prediction problems in non-linear non-Gaussian process [38]. In comparison with standard approximation methods in class of stochastic filtering, such as the popular Extended Kalman Filter, the main advantage of particle methods is that they do not rely on any local linearization technique or any crude functional approximation [39]. The price that must be paid for this flexibility is computationally expensive. However, thanks to the availability of everincreasing computational power, these methods are already used in real-time applications appearing 
in fields as diverse as chemical engineering, computer vision, financial econometrics, target tracking and robotics [40]. This is why we use the particle filter approach to estimate in the real-time the crack depth of the system at discrete inspection times.

The used particle filter technique is quite simple and is similar to simulation approach of Zio et al. in $[9,12]$. Because the focus of the paper is not computation methods, we do not explain the principle of the considered particle filter. Interested reader can refer to $[9,12]$ for the detail development. Instead of this, we present explicitly the particle filter algorithm that does not be introduced in these papers. The algorithm uses the prior distribution derived from (2.5) as the importance function, and the deterministic re-sampling method [41] to limit the inherent degeneracy problem in the particle filter approach. As such, the real-time state estimation procedure, given the sequence of measurements $\mathbf{Z}_{T_{0: i}}=\left\{z_{T_{0}}, z_{T_{1}}, \ldots, z_{T_{i}}\right\}$, can be resumed by Algorithm 1.

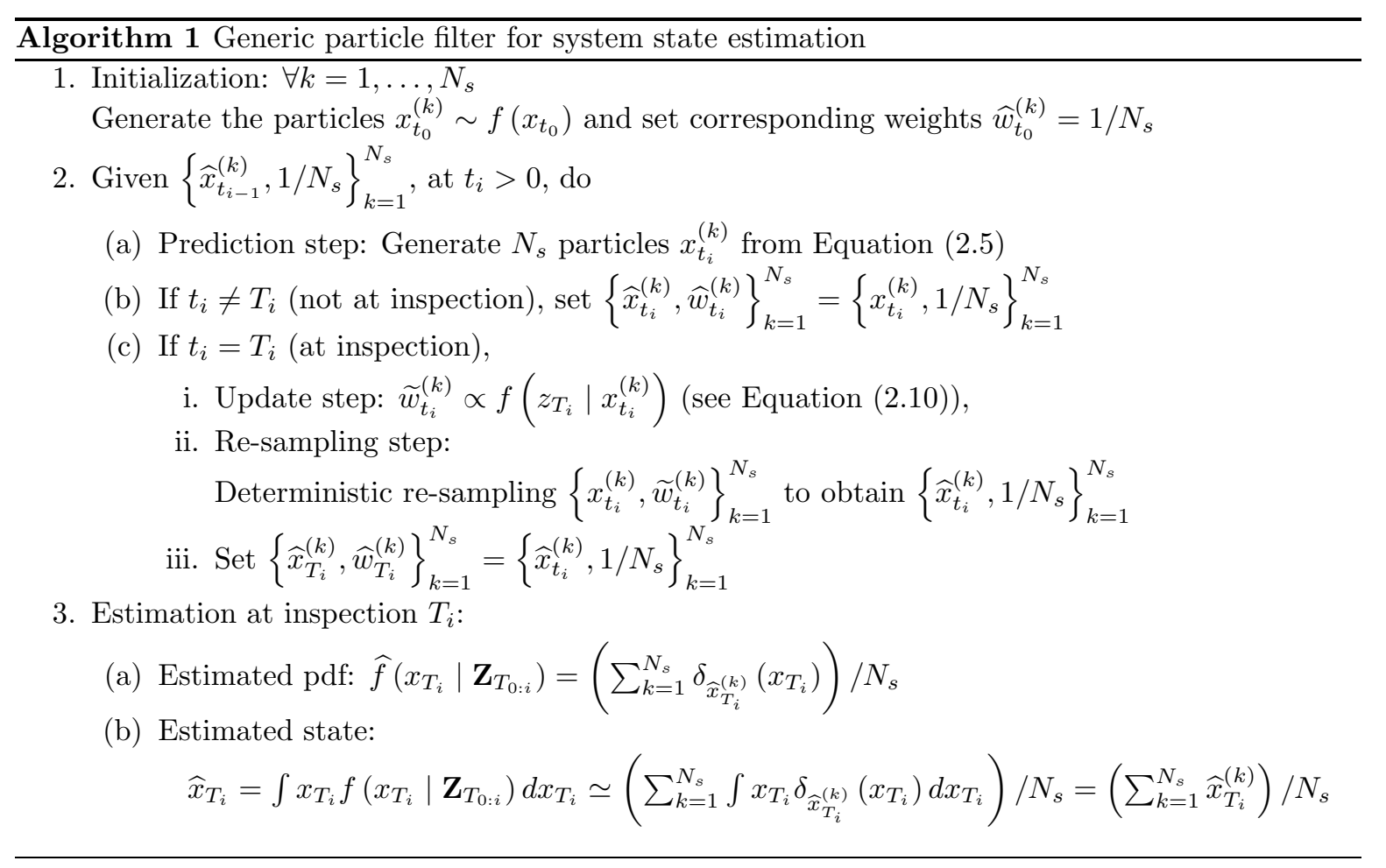

To illustrate the robustness of the proposed algorithm, consider again the system defined in the example of Section 2 with $\alpha=0.003$. The parameters of the measurement model are $\beta_{0}=0.06$, $\beta_{1}=1.25$ and $\sigma_{v}=0.5$. The samples number is $N_{s}=3000$, and the inspection is periodic with a 
period $\Delta T=2$. Sub-figure 4a shows the evolution of operating environment and the relative standard deviation of the measurement (i.e. $\epsilon_{t_{i}}=\frac{\left\|z_{t_{i}}-x_{t_{i}}\right\|}{x_{t_{i}}} \times 100$ ). Sub-figure $4 \mathrm{~b}$ shows the estimated results of degradation state at inspection times. We can remark that the error between the estimated state and

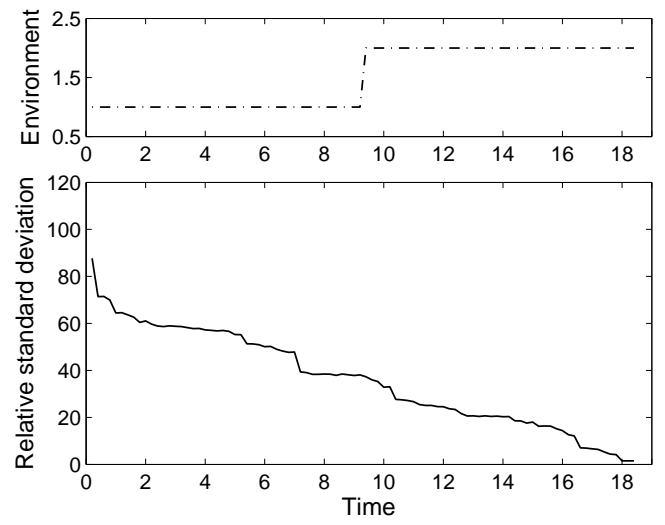

(a) Environment evolution and measurement error

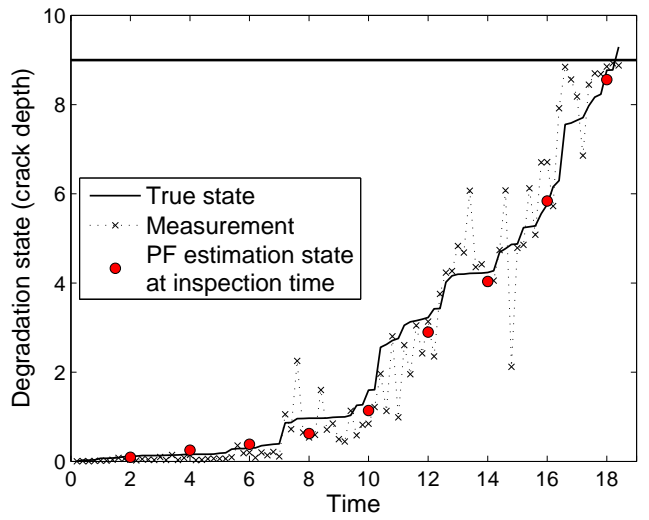

(b) Degradation evolution and state estimation

Figure 4: State estimation by generic particle filter algorithm

the true degradation state is unavoidable because the degradation is estimated by its expected value conditional on the sequence of noisy measurements, and the PF algorithm is just an approximation of the optimal Bayesian estimate. However, the estimated state can still approximate the true degradation path more closely compared to the measured data.

\subsection{Monte Carlo simulation for maintenance optimization}

In this subsection, the Monte Carlo simulation is used to approximate the optimal solutions of the proposed adaptive maintenance strategies. Thus, the long run expected maintenance cost rate given from Equation (3.1) is rewritten to

$$
C_{\infty}=\frac{E[C(S)]}{E[S]}=\lim _{N \rightarrow \infty} \frac{\frac{1}{N} \sum_{n=1}^{N} C\left(S_{n}\right)}{\frac{1}{N} \sum_{n=1}^{N} S_{n}} \simeq \frac{\sum_{n=1}^{N_{h}} C\left(S_{n}\right)}{\sum_{n=1}^{N_{h}} S_{n}}, \text { with } N_{h} \text { high enough, }
$$

where $N_{h}$ is the number of simulation histories and $S_{n}$ is the length of the first renewal cycle of the $n$-th history. The simulation procedure to obtain the long run expected maintenance cost rate $C_{\infty}$ according to Equation (4.1) is given by Algorithm 2. 


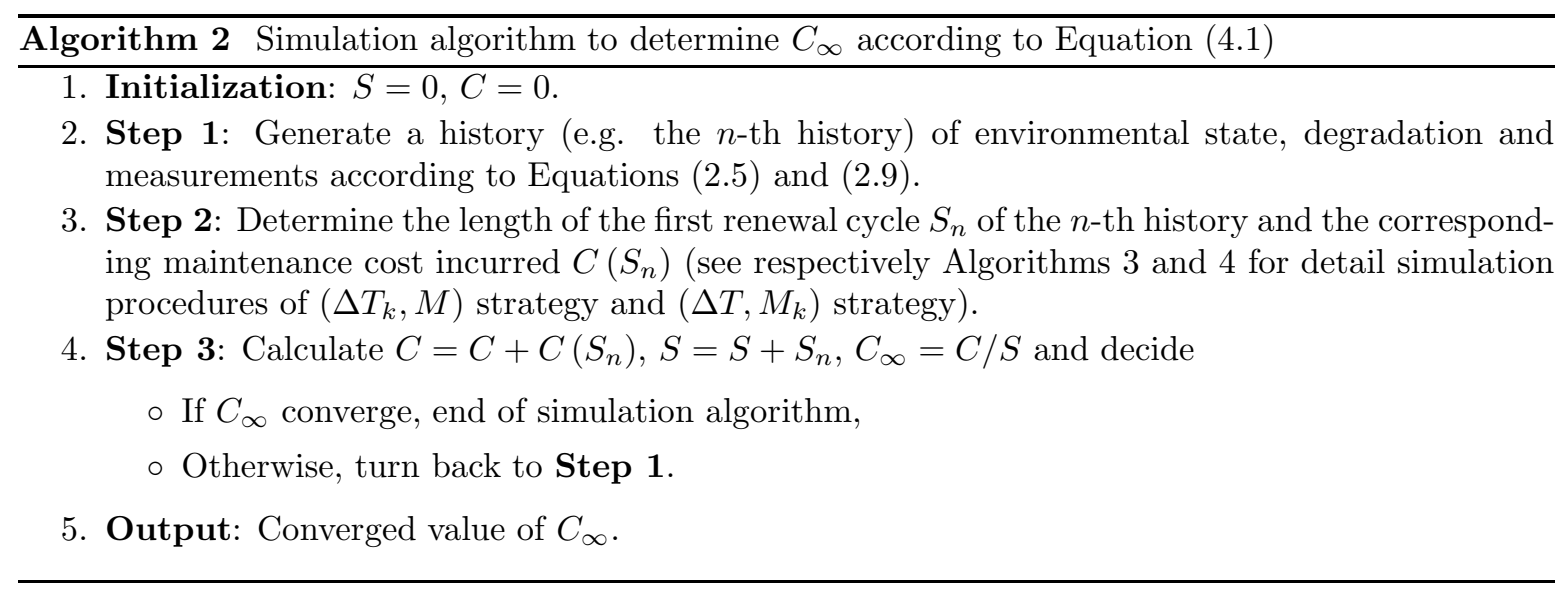

For numerical illustrations in the remainder of this section, the system and the measurement model described in the example of Subsection 4.1 is recalled. The measurement error is re-chosen by $\sigma_{v}=0.4$ for a clearer illustration. The samples number is $N_{s}=3000$, the number of simulated histories is $N_{h}=4000$ (the value of $C_{\infty}$ is converged when $N_{h}=4000$ ). Finally, the intervention costs are chosen by $C_{i}=8, C_{p}=50, C_{c}=100$ and $C_{d}=25$.

\subsubsection{Numerical solution for $\left(\Delta T_{k}, M\right)$ strategy}

This section shows how to obtain the length of the renewal cycle $S_{n}^{\Delta T_{k}, M}$ and the maintenance cost $C^{\Delta T_{k}, M}\left(S_{n}^{\Delta T_{k}, M}\right)$ (i.e., step 2 of Algorithm 2) by applying the decision rule of $\left(\Delta T_{k}, M\right)$ strategy on the $n$-th history. The simulation procedure is described by Algorithm 3. Algorithms 2 and 3 provide a complete numerical evaluation of the long-run expected maintenance cost rate $C_{\infty}^{\Delta T_{k}, M}\left(\Delta T_{1}, \ldots, \Delta T_{m}, M\right)$ of $\left(\Delta T_{k}, M\right)$ strategy. With the chosen data set, the time to compute $C_{\infty}^{\Delta T_{k}, M}\left(\Delta T_{1}, \ldots, \Delta T_{m}, M\right)$ for a specific set of $\left(\Delta T_{1}, \ldots, \Delta T_{m}, M\right)$ is less than 4 minutes. Given $C_{\infty}^{\Delta T_{k}, M}\left(\Delta T_{1}, \ldots, \Delta T_{m}, M\right)$, many optimization methods can be introduced to optimize $\left(\Delta T_{k}, M\right)$ strategy. Here, since the aim of the paper is not to develop new methods of optimization, a classical iterative optimization method (i.e. cyclic coordinate descent algorithm [42]) has been used. Figures 5, 6 and 7 show the cost rate $C_{\infty}^{\Delta T_{k}, M}\left(\Delta T_{1}, \Delta T_{2}, M\right)$ for the selected data set when one of decision parameters is fixed at its optimal value. The sub-figures on the left and on the right represent respectively the shape and the iso-level cost curves of $C_{\infty}^{\Delta T_{k}, M}\left(\Delta T_{1}, \Delta T_{2}, M\right)$. The convexity of the cost surface indicates the existence of an optimum setting of $\Delta T_{1}, \Delta T_{2}$ and $M$. In fact, the optimal values of decision 


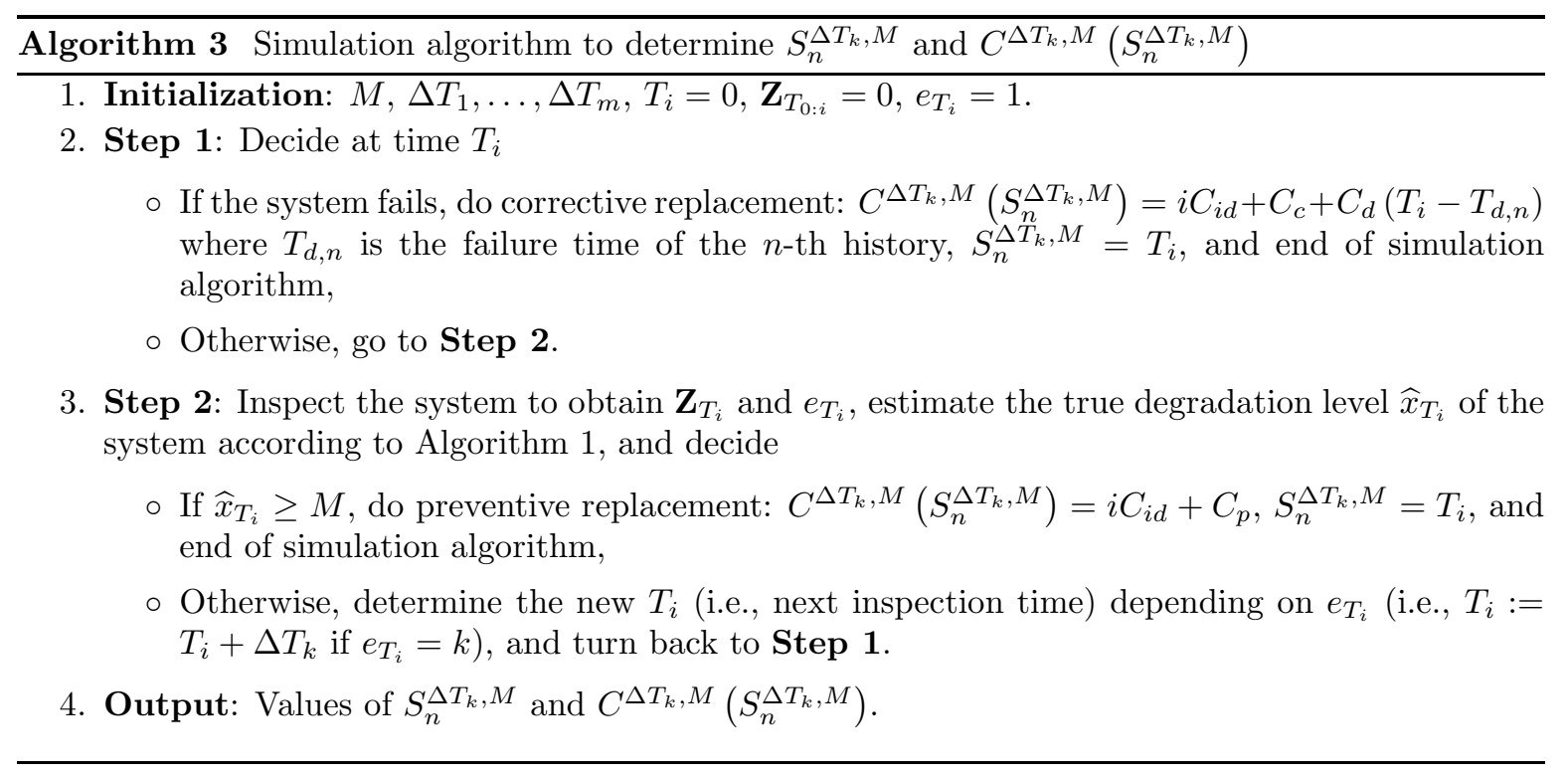

variables are $\Delta T_{1, o p t}=15, \Delta T_{2, o p t}=4$ and $M_{o p t}=5$ that correspond to an optimal cost rate $C_{\infty}^{\Delta T_{k}, M}\left(\Delta T_{1, o p t}, \Delta T_{2, o p t}, M_{o p t}\right)=5.6354$
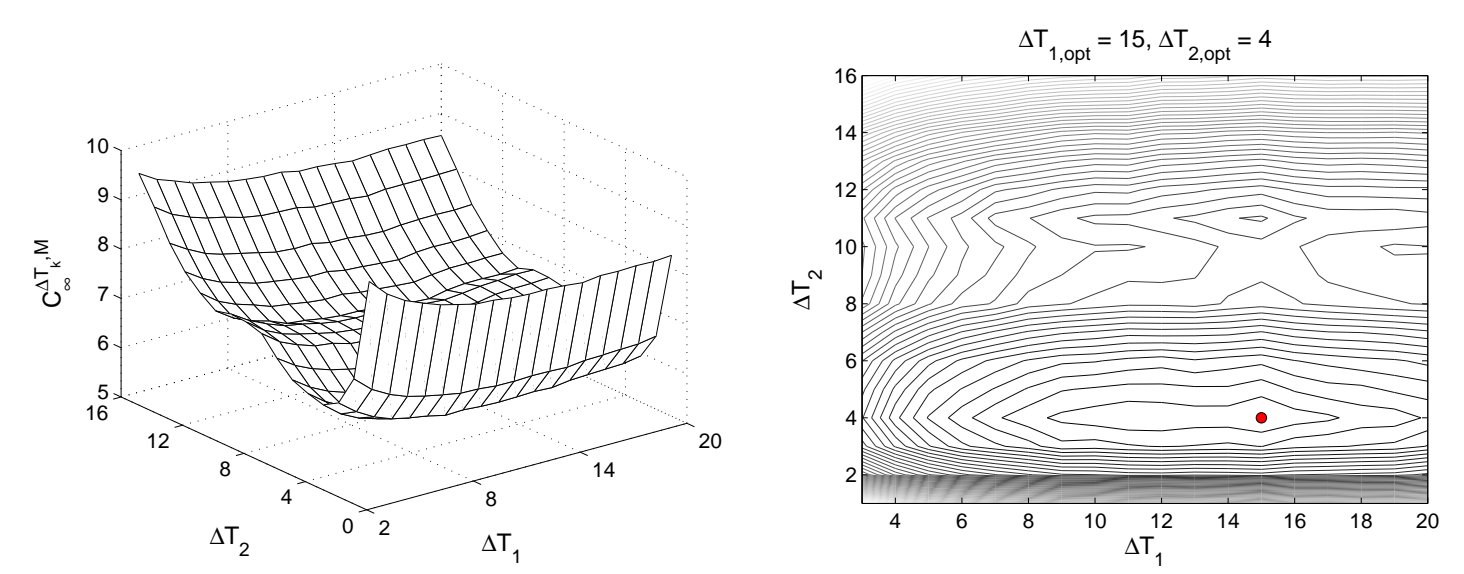

Figure 5: Expected maintenance cost rate $C_{\infty}^{\Delta T_{k}, M}\left(\Delta T_{1}, \Delta T_{2}, M\right)$ when $M$ is fixed at $M_{o p t}=5$

\subsubsection{Numerical solution for $\left(\Delta T, M_{k}\right)$ strategy}

In this section, we show how to obtain the length of the renewal cycle $S_{n}^{\Delta T, M_{k}}$ and the maintenance cost $C^{\Delta T, M_{k}}\left(S_{n}^{\Delta T, M_{k}}\right)$ (i.e., step 2 of Algorithm 2) by applying the decision rule of $\left(\Delta T, M_{k}\right)$ strategy on the $n$-th history. The simulation procedure is described by Algorithm 4. Algorithms 

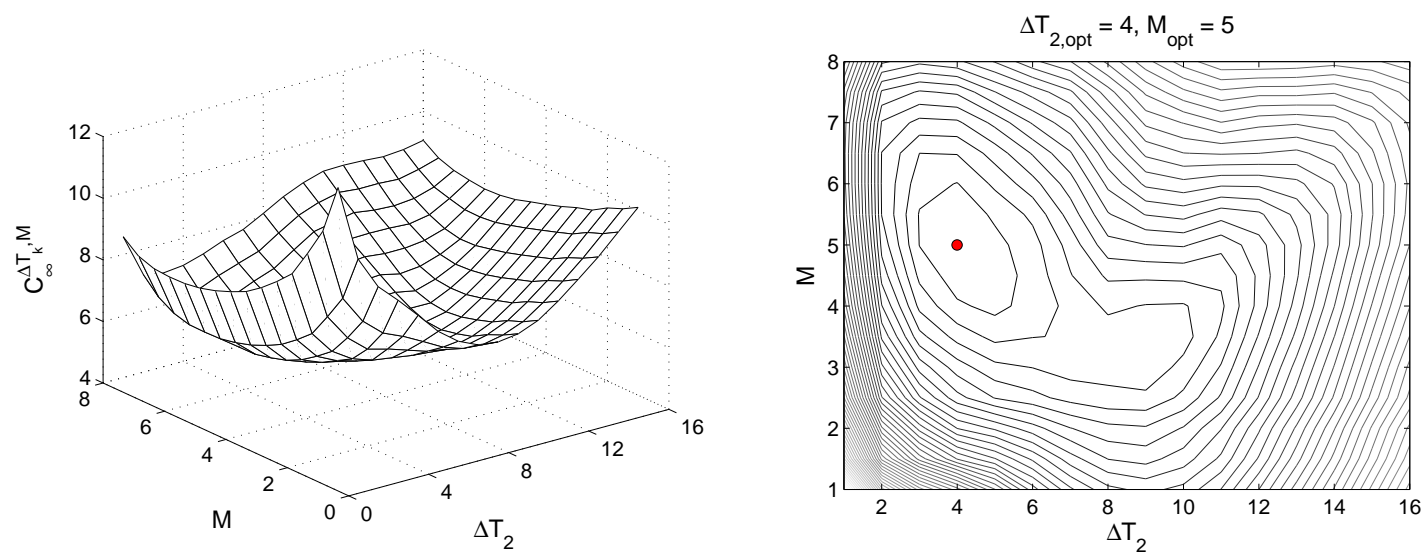

Figure 6: Expected maintenance cost rate $C_{\infty}^{\Delta T_{k}, M}\left(\Delta T_{1}, \Delta T_{2}, M\right)$ when $\Delta T_{1}$ is fixed $\Delta T_{1, \text { opt }}=15$
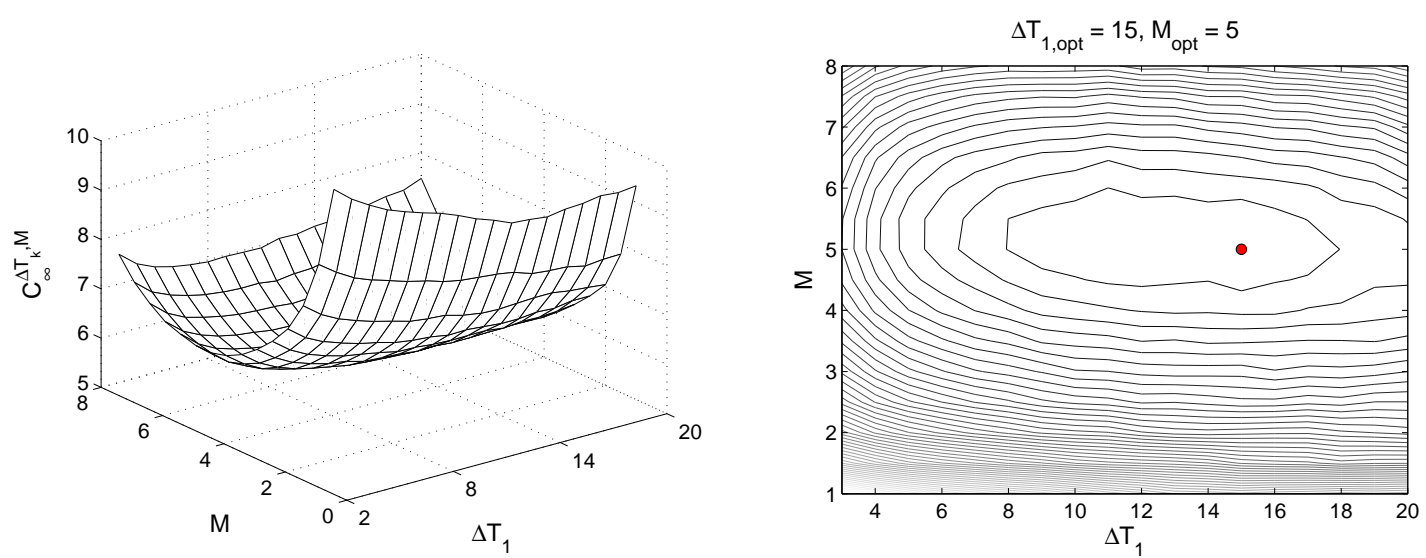

Figure 7: Expected maintenance cost rate $C_{\infty}^{\Delta T_{k}, M}\left(\Delta T_{1}, \Delta T_{2}, M\right)$ when $\Delta T_{2}$ is fixed at $\Delta T_{2, \text { opt }}=4$

2 and 4 provide a complete numerical evaluation of the long-run expected maintenance cost rate $C_{\infty}^{\Delta T, M_{k}}\left(\Delta T, M_{1}, \ldots, M_{m}\right)$ of $\left(\Delta T_{k}, M\right)$ strategy. With the chosen data set, the time to compute $C_{\infty}^{\Delta T, M_{k}}\left(\Delta T, M_{1}, \ldots, M_{m}\right)$ for a specific set of $\left(\Delta T, M_{1}, \ldots, M_{m}\right)$ is about 4 minutes. As the same reason mentioned in the previous section, the classical cyclic coordinate descent algorithm has been used to optimize the $\left(\Delta T, M_{k}\right)$ strategy. Figures 8,9 and 10 show the cost rate $C_{\infty}^{\Delta T, M_{k}}\left(\Delta T, M_{1}, M_{2}\right)$ for the selected data set when one of decision parameters is fixed at its optimal value. The sub-figures on the left and on the right represent respectively the shape and the iso-level cost curves of $C_{\infty}^{\Delta T, M_{k}}\left(\Delta T, M_{1}, M_{2}\right)$. The convex cost surface indicates the existence of an optimum setting of $\Delta T, M_{1}$ and $M_{2}$. In fact, the optimal cost rate is $C_{\infty}^{\Delta T, M_{k}}\left(\Delta T_{\text {opt }}, M_{1, \text { opt }}, M_{2, \text { opt }}\right)=5.1607$ that corresponds to the following optimal 


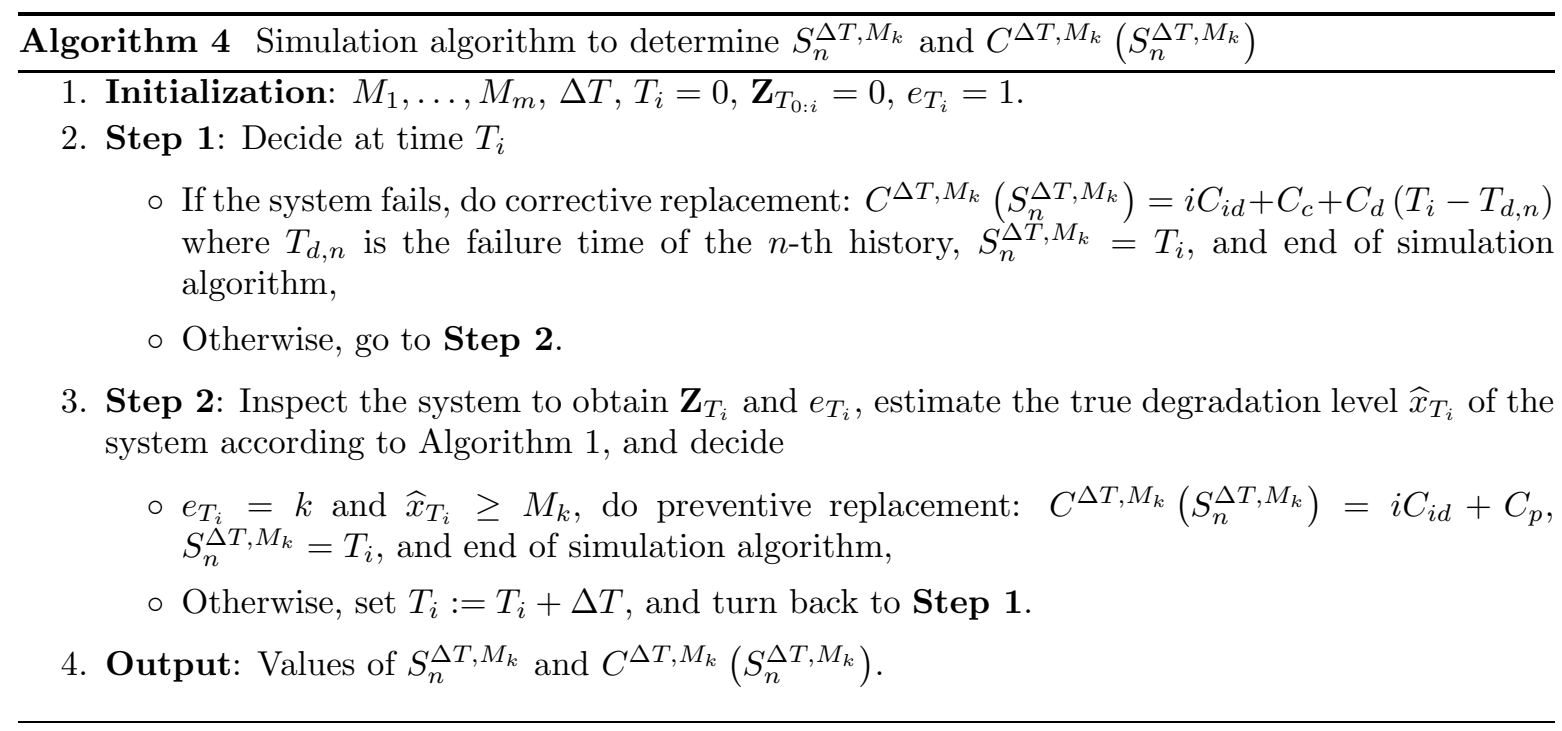

value of decision variables $\Delta T_{o p t}=8, M_{1, \text { opt }}=7.5$ et $M_{2, \text { opt }}=1.5$. This optimal expected maintenance cost rate is lower than the one of $\left(\Delta T_{k}, M\right)$ strategy $\left(C_{\infty}^{\Delta T, M_{k}}\left(\Delta T_{\text {opt }}, M_{1, \text { opt }}, M_{2, \text { opt }}\right)=5.1607\right.$ vs. $\left.C_{\infty}^{\Delta T_{k}, M}\left(\Delta T_{1, o p t}, \Delta T_{2, o p t}, M_{o p t}\right)=5.6354\right)$. However, we cannot conclude that the $\left(\Delta T, M_{k}\right)$ strategy is always more profitable than the $\left(\Delta T_{k}, M\right)$ strategy, because the cost saving depends closely on the intervention costs, as well as on the dynamic characteristics of system. The next section deals with this problem to point out the adequate applicable conditions of each adaptive maintenance strategy.
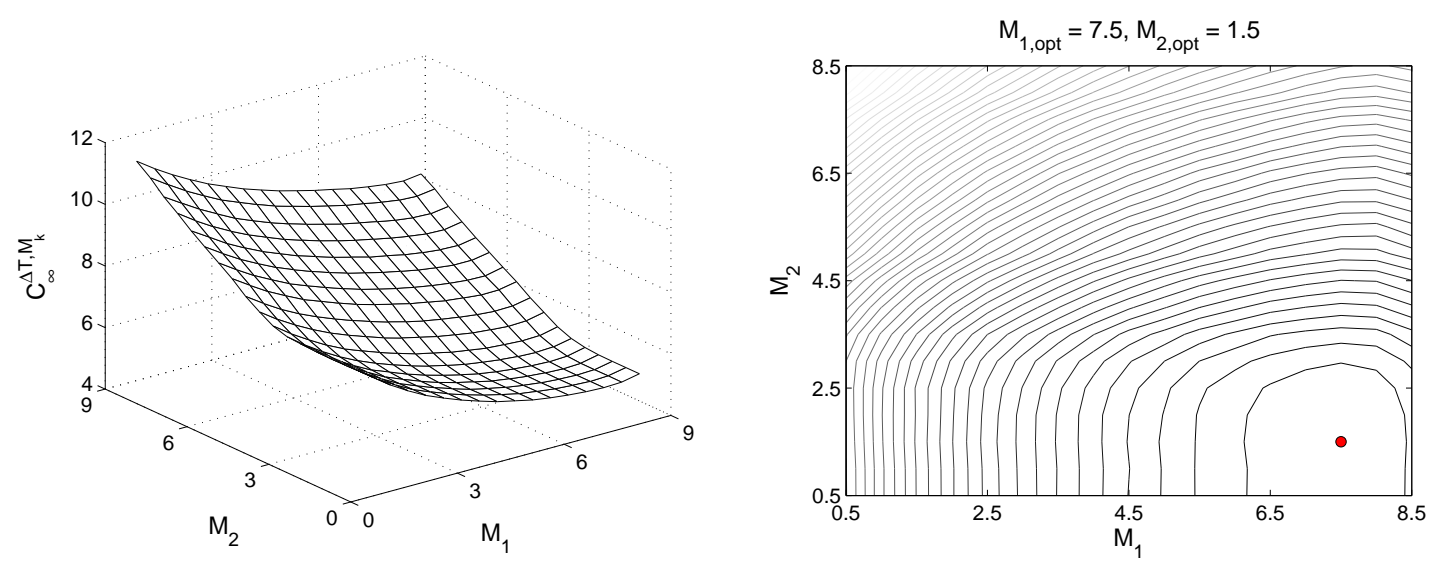

Figure 8: Expected maintenance cost rate $C_{\infty}^{\Delta T, M_{k}}\left(\Delta T, M_{1}, M_{2}\right)$ when $\Delta T$ is fixed at $\Delta T_{o p t}=8$ 

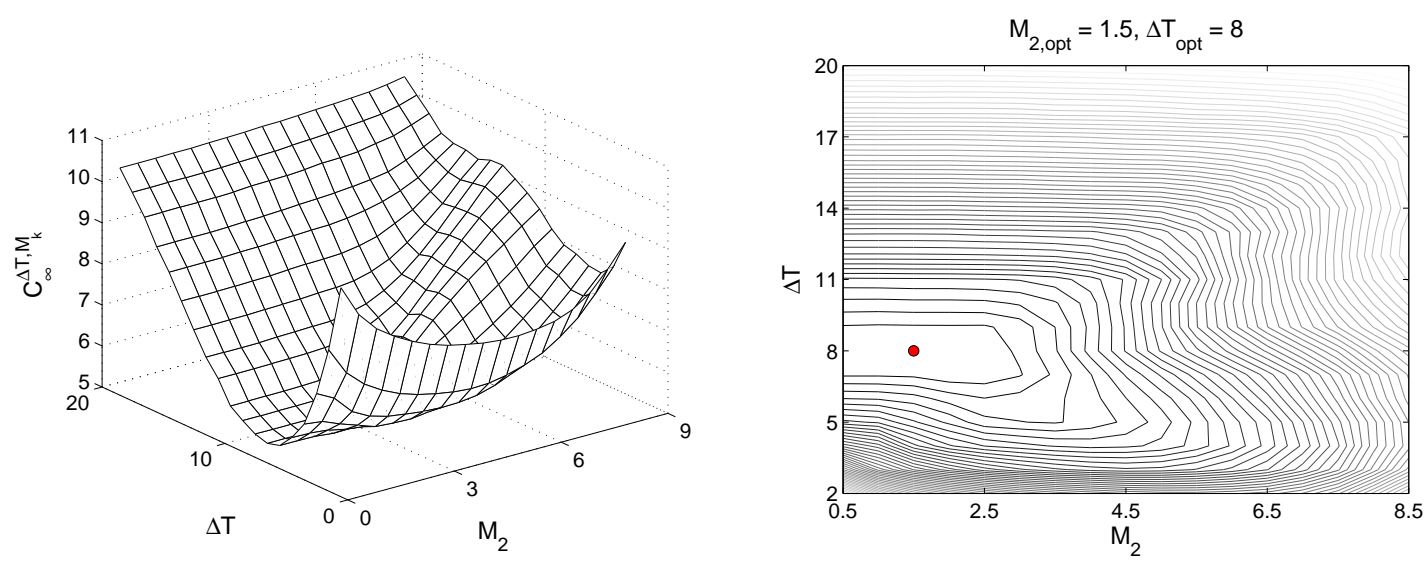

Figure 9: Expected maintenance cost rate $C_{\infty}^{\Delta T, M_{k}}\left(\Delta T, M_{1}, M_{2}\right)$ when $M_{1}$ is fixed at $M_{1, \text { opt }}=7.5$
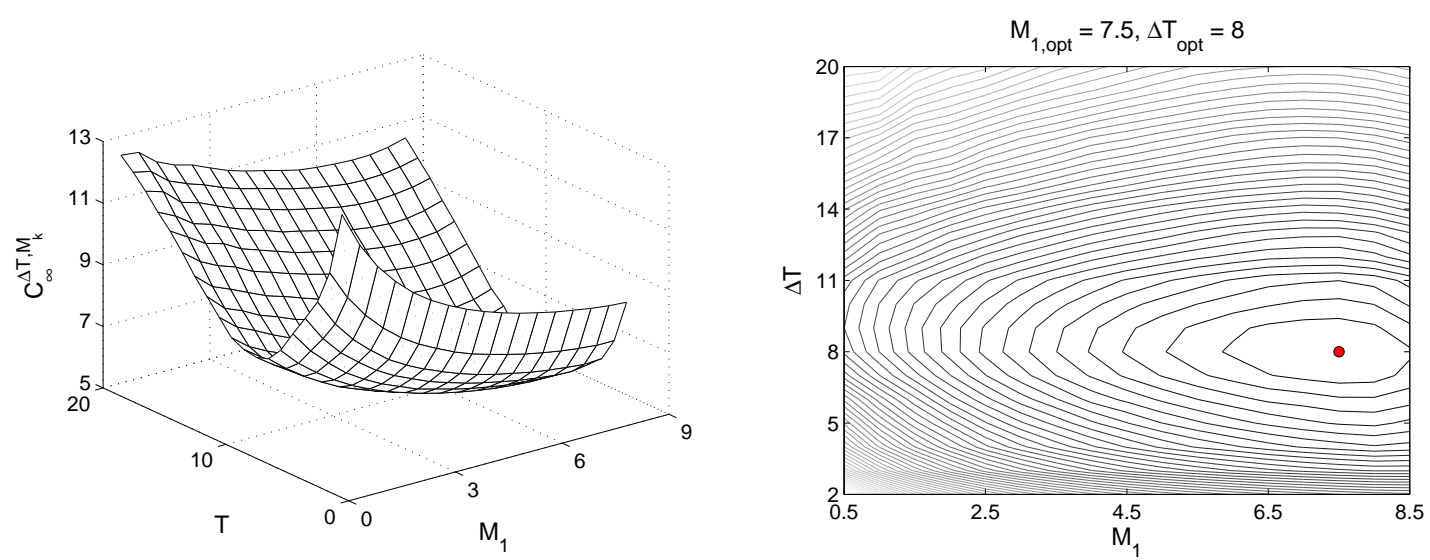

Figure 10: Expected maintenance cost rate $C_{\infty}^{\Delta T, M_{k}}\left(\Delta T, M_{1}, M_{2}\right)$ when $M_{2}$ is fixed at $M_{2, \text { opt }}=1.5$

\section{Performance and robustness analysis of the adaptive maintenance strategies}

The focus of this section is to study the performance and the robustness of $\left(\Delta T_{k}, M\right)$ strategy and $\left(\Delta T, M_{k}\right)$ strategy by comparing one to each other and to the classical $(\Delta T, M)$ strategy through numerical examples. We shall analyze the influence of the maintenance actions costs, the characteristics of stressful operating environment, and the impact of estimation error made on parameters of degradation process on the optimal values of the decision variable and of the optimal expected maintenance cost rate of the considered strategies. Analyzing the optimal cost rate allows us to assess the performance and the robustness of each maintenance strategy, and investigating the evolution of optimal decision variables gives a trend to adjust the strategies towards their optimal solution. 


\subsection{Sensitivity to the maintenance costs}

To analyze the sensitivity of the maintenance strategies to the maintenance costs, we vary one of intervention costs (i.e. preventive replacement cost $C_{p}$, inspection cost $C_{i}$, or the cost rate for the system inactivity $C_{d}$ ), fix the other costs and investigate the evolution of the optimal values of decision variables and of the optimal expected maintenance cost rate. The corrective replacement cost does not cause so much difference for the relative performance of the considered strategies, so we can fix it at $C_{c}=100$. The study is based on the same system defined in the numerical examples of Subsection 4.2. It is noted that the 2-state Markovian environment is chosen just for the best analysis, but a more complex environment (e.g., more than 2 states with different transition speeds) is easily obtained by modifying the dimension of the transition matrix $\mathcal{T}_{\alpha}$ as well as its transition probabilities (see Equation $(2.3))$.

\subsubsection{Sensitivity to preventive replacement cost}

To have an unbiased study of the influence of preventive replacement cost on the evolution of the optimal decision variables and of the optimal expected cost rate for considered maintenance strategies, we choose $C_{c}=100, C_{i}=4, C_{d}=25$ and we vary the preventive replacement cost $C_{p}$ in a large interval from $C_{i}$ to $C_{c}$ with the cost step equal to 1 . The results for this case are showed as in Figure 11. Sub-figures $11 \mathrm{a}$ and $11 \mathrm{~b}$ represent respectively the evolutions of the optimal decision variables of $\left(\Delta T_{k}, M\right)$ strategy and $\left(\Delta T, M_{k}\right)$ strategy, and Sub-figure 11c shows the evolutions of the optimal expected maintenance cost rate of the three considered strategies. The optimal values of inspection periods of $\left(\Delta T_{k}, M\right)$ strategy and $\left(\Delta T, M_{k}\right)$ strategy are more or less constant with respect to $C_{p}$, while the optimal values of preventive replacement threshold increase with $C_{p}$. This means that few preventive replacements are performed when their cost is high and this also reflects the fundamental nature of CBM strategy where the preventive replacement decision is based on the degradation level of the system. This remark is similar to the one of $\left(\Delta T_{k}, M\right)$ strategy studied by Huynh et al. in [6], but extending to the maintenance strategy with multi-periods of inspection or multi-thresholds of preventive replacement. Since the latter can adapt to the variation of operating environment to adjust more finely the decision variables, it is always more profitable than the former (see Sub-figure 11c).

Sub-figure $11 \mathrm{c}$ also shows that the $\left(\Delta T, M_{k}\right)$ strategy can lead to more saving in maintenance cost 

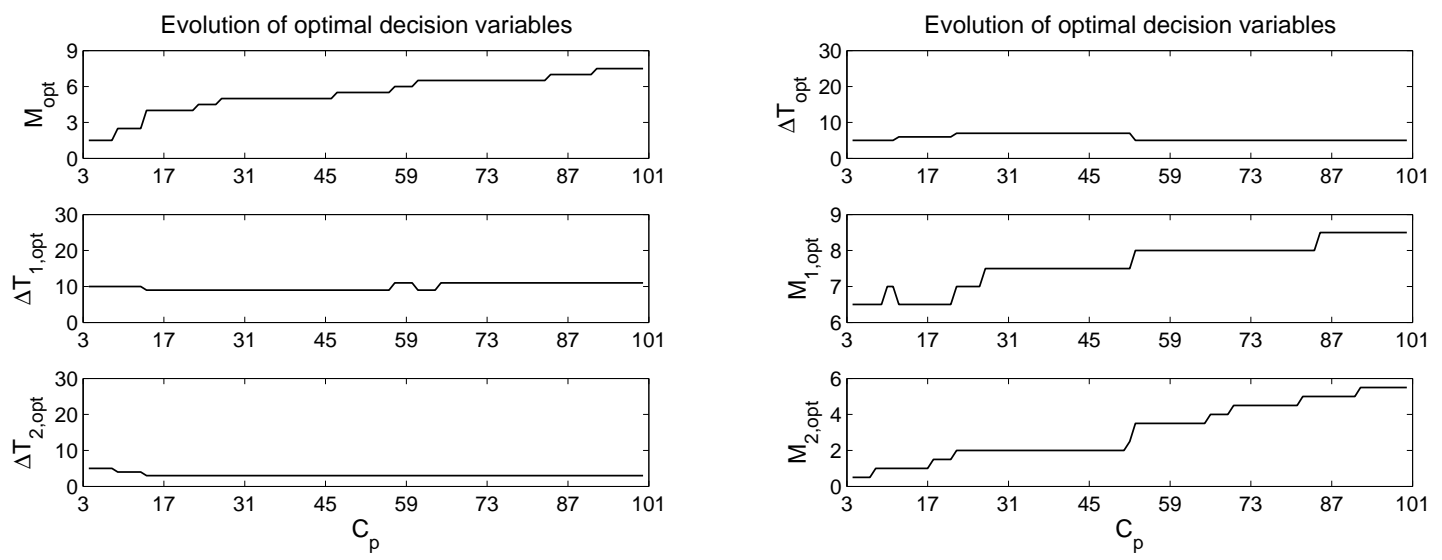

(a) $\left(\Delta T_{k}, M\right)$ strategy

(b) $\left(\Delta T, M_{k}\right)$ strategy

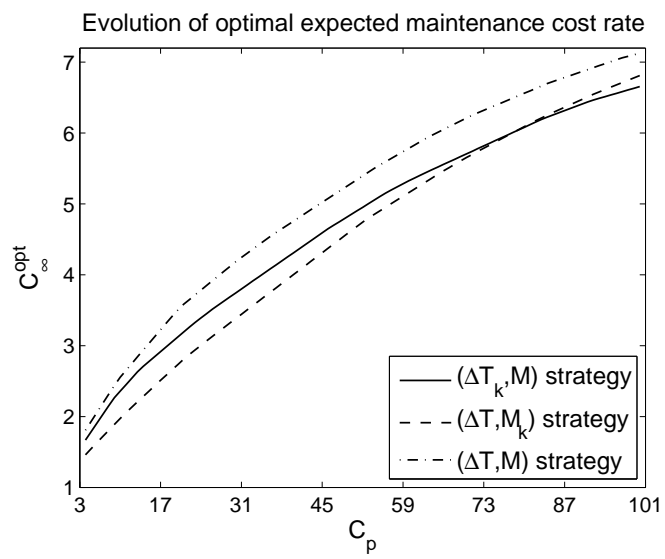

(c) Adaptive strategies vs. $(\Delta T, M)$ strategy

Figure 11: Varied preventive replacement cost

than the $\left(\Delta T_{k}, M\right)$ strategy when the preventive replacement is not costly, because in this situation making an adaptation decision based on the degradation level seems to be more suitable than the one based on time. But, when the preventive replacement $\operatorname{cost} C_{p}$ is higher and comes close to the corrective replacement cost $C_{c}$, the $\left(\Delta T, M_{k}\right)$ strategy loses its interest and becomes less profitable. The reason is that the strategy can accept the system failures in this case, the inspection periods become then an important factor that keep the system downtime at a minimal value. In this consideration, the $\left(\Delta T_{k}, M\right)$ strategy that allows the time-based adaptation is more advantageous than the $\left(\Delta T, M_{k}\right)$ strategy. 


\subsubsection{Sensitivity to inspection cost}

To investigate the sensitivity of the optimal decision variables and the optimal expected maintenance cost rate to inspection cost, we choose the set of maintenance cost as follows. The preventive replacement cost is chosen by $C_{p}=75$, as in Sub-figure 11c the optimal cost rates of $\left(\Delta T_{k}, M\right)$ strategy and $\left(\Delta T, M_{k}\right)$ strategy are identical at this value. The corrective replacement cost and the cost rate of system inactivity are fixed at $C_{c}=100, C_{d}=25$. Finally, we vary the inspection cost $C_{i}$ from 2 to $C_{p}$ with the cost step equal to 1 . The evolutions of the optimal decision variables and the optimal expected maintenance cost rate for this case study are shown respectively in Sub-figures 12a, 12b and 12c. As in the $(\Delta T, M)$ strategy considered by Huynh et al. in [6], the optimal value of inspection periods in the $\left(\Delta T_{k}, M\right)$ strategy and the $\left(\Delta T, M_{k}\right)$ strategy tends to increase with the increasing of inspection cost in order to avoid more frequent inspections. However, their preventive replacement thresholds are not always decreasing as in $(\Delta T, M)$. In fact, we can see in the Sub-figures 12a and $12 \mathrm{~b}$ that $M_{\text {opt }}$ and $M_{2, o p t}$ increase slightly when $C_{i}$ is set at a high value. This can be explained by the fact that the inspection periods are very large in this situation, the replacement (either preventive or corrective) is almost always triggered at the first inspection time. The optimal values of $M_{\text {opt }}$ and $M_{2, o p t}$ are then adjusted higher to limit the number of replacements. This is why the $\left(\Delta T_{k}, M\right)$ strategy and $\left(\Delta T, M_{k}\right)$ strategy are more flexible than the $(\Delta T, M)$ strategy, and not surprising, they are always more efficient (see Sub-figure 12c).

We can also remark that the $\left(\Delta T_{k}, M\right)$ strategy becomes more advantageous than the $\left(\Delta T, M_{k}\right)$ strategy when the inspection cost is high. The reason is that the $\left(\Delta T_{k}, M\right)$ strategy can better manage the inspection number by adapting the inspection periods to the environmental state. Furthermore, since the system downtime is more important under this configuration, a time-based adaptation approach as in the $\left(\Delta T_{k}, M\right)$ strategy is more relevant than a condition-based approach as in the $\left(\Delta T, M_{k}\right)$ strategy.

\subsubsection{Sensitivity to the cost rate of system inactivity}

In the same way as in previous studies, to examine the impact of the cost rate of system inactivity $C_{d}$ on the characteristics of the proposed maintenance strategies, we fix $C_{c}=100, C_{i}=4, C_{p}=75$, and we vary $C_{d}$ in a large interval from 5 to 150 with the cost step equal to 1 . Figure 13 reports the 

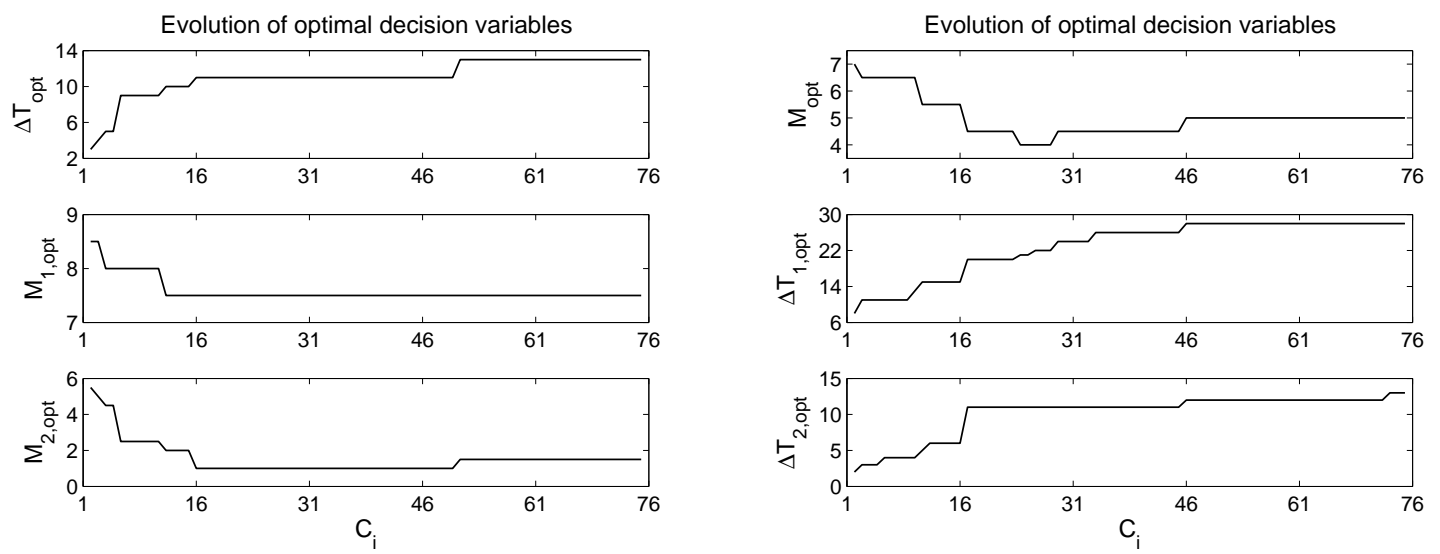

(a) $\left(\Delta T_{k}, M\right)$ strategy

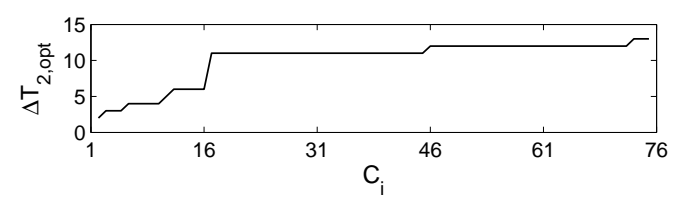

(b) $\left(\Delta T, M_{k}\right)$ strategy

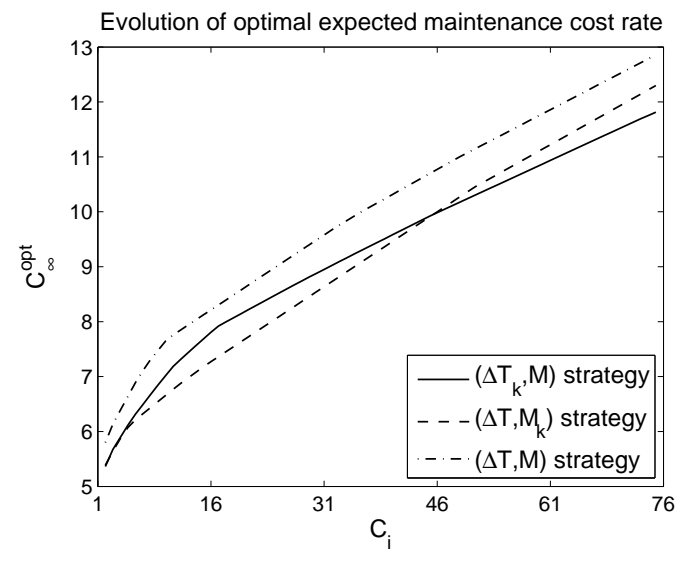

(c) Adaptive strategies vs. $(\Delta T, M)$ strategy

Figure 12: Varied inspection cost

results. One obtains in this case study a similar phenomenon as in the $(\Delta T, M)$ strategy considered in [6]: the optimal preventive replacement thresholds are almost constant, while the optimal inspection periods decrease with $C_{d}$ to cut short the system downtime (see Sub-figures 13a and 13b). This means that to adapt these strategies to the variation of $C_{d}$, it suffices to adjust the inspection periods because they are the most sensitive decision variables to this variation.

From Sub-figure 13c, one notes firstly the benefit of the adaptive decisions following the current state of the variable environment: the $\left(\Delta T_{k}, M\right)$ strategy and the $\left(\Delta T, M_{k}\right)$ strategy can almost always lead to a substantial saving in maintenance cost compared to $(\Delta T, M)$ strategy. Moreover, since the 

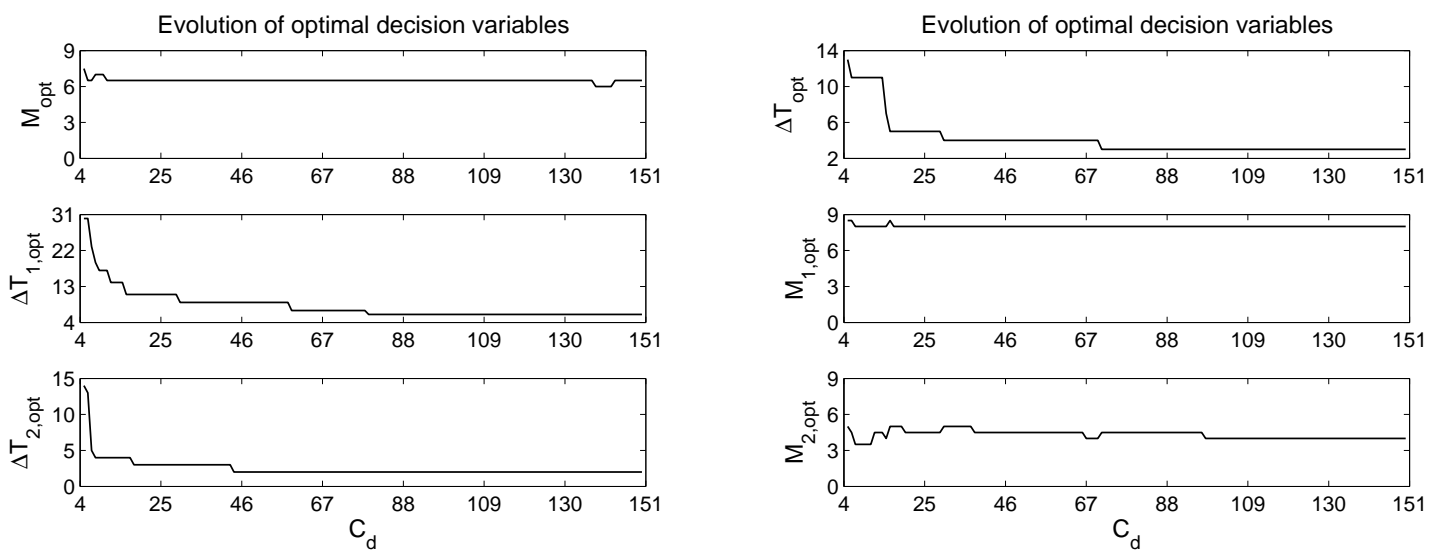

(a) $\left(\Delta T_{k}, M\right)$ strategy

(b) $\left(\Delta T, M_{k}\right)$ strategy

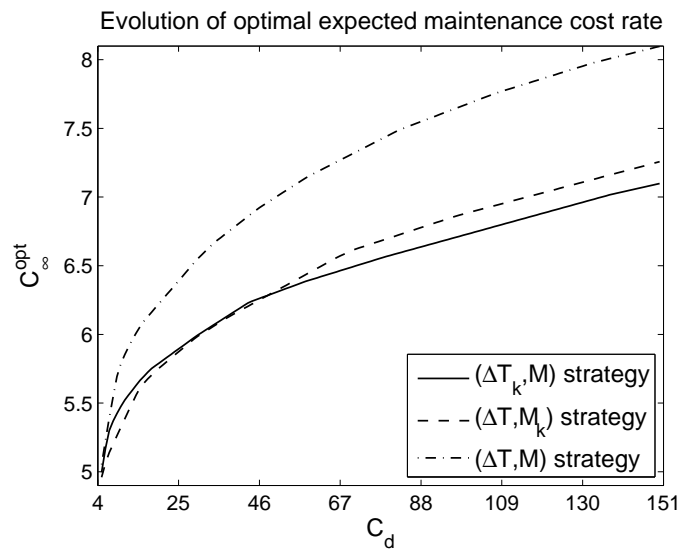

(c) Adaptive strategies vs. $(\Delta T, M)$ strategy

Figure 13: Varied cost rate of system inactivity

$\left(\Delta T_{k}, M\right)$ strategy reduces better the system downtime compared to the $\left(\Delta T, M_{k}\right)$ strategy, it is hence more profitable when the inactivity cost rate becomes more important.

\subsection{Sensitivity to the characteristics of operating environment}

The main differences among the adaptive maintenance strategies proposed in this paper and the classical one are the abilities to adapt to the variation of operating environment. It is, therefore, reasonable to study the sensitivity of the maintenance strategies to environment characteristics. The studies are always based on the system defined in Subsection 5.1, however for this time, the characteristics of operating environment shall be varied. The maintenance costs are chosen by $C_{c}=100$, 
$C_{p}=50, C_{i}=8$ and $C_{d}=25$.

\subsubsection{Sensitivity to the stress level of environment}

We consider a 2 -state Markovian environment (i.e., $\Xi=\{1,2\}, e_{t_{0}}=1$ ) whose the transition speed is defined by $\alpha=0.003$, (i.e., low speed - see Sub-figure 1a). We fix the stress level of the normal state $e_{t_{i}}=1$ at $\gamma_{1}=0$, and we vary the stress level of the stressful state $e_{t_{i}}=2$ in the range of values $\gamma_{2}=2,3,4,5,6,7$. As such, the difference between the stress levels of these 2 states is $\Delta \gamma=\gamma_{2}-\gamma_{1}=2,3,4,5,6,7$. We shall study here the evolutions of the optimal decision variables and of the optimal expected maintenance cost rate of $\left(\Delta T_{k}, M\right)$ strategy and $\left(\Delta T, M_{k}\right)$ strategy according to this difference. Figure 14 shows the results of the case study.

The evolutions of optimal decision variables of $\left(\Delta T_{k}, M\right)$ strategy are showed in Sub-figure 14a. We can note that the values of $M_{o p t}$ and $\Delta T_{1, o p t}$ are more or less constant, while only the value of $\Delta T_{2, o p t}$ strictly decreases with respect to $\Delta \gamma$. This is because only the stress level of the state $e_{2}=2$ varies, and the decreasing of $\Delta T_{2, \text { opt }}$ aims to monitor more frequently the system health in order to limit the failures number of system and to shorten its inactivity interval. Sub-figure 14b reports the evolutions of optimal decision variables of $\left(\Delta T, M_{k}\right)$ strategy. The values of $\Delta T_{\text {opt }}$ and $M_{2, \text { opt }}$ decrease in order to monitor more frequently the system health and/or to guarantee a larger safety zone for the system when the environment becomes more and more stressful. These adjustments allows to cut short the system downtime as well as limit the number of failures. On the contrary, the increasing of $M_{1, o p t}$ aims to create a trade-off between intervention actions to give best profit for the strategy. From these analyses, it seems that the $\left(\Delta T, M_{k}\right)$ strategy is more flexible than the $\left(\Delta T_{k}, M\right)$ strategy when the stress level of operating environment varies. The $\left(\Delta T, M_{k}\right)$ strategy can hence lead to more saving than the others (see Sub-figure 14c). In fact, both adaptive strategies are profitable than the $(\Delta T, M)$ strategy, and the gain increase with respect to the stress level. More precisely, the higher the difference of stress levels, the more the gain of the $\left(\Delta T, M_{k}\right)$ strategy is larger than the $\left(\Delta T_{k}, M\right)$ strategy. This result can be explained by the consideration that the speed and the variance of degradation process increase with respect to the stress level, so making an adaptation decision based on the current degradation level as in the $\left(\Delta T, M_{k}\right)$ strategy is more efficient than an adaptation decision based on time as in the $\left(\Delta T_{k}, M\right)$ strategy. 

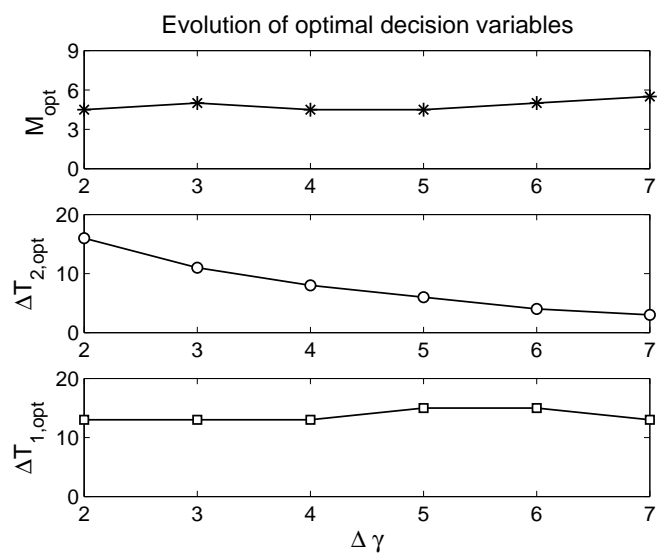

(a) $\left(\Delta T_{k}, M\right)$ strategy
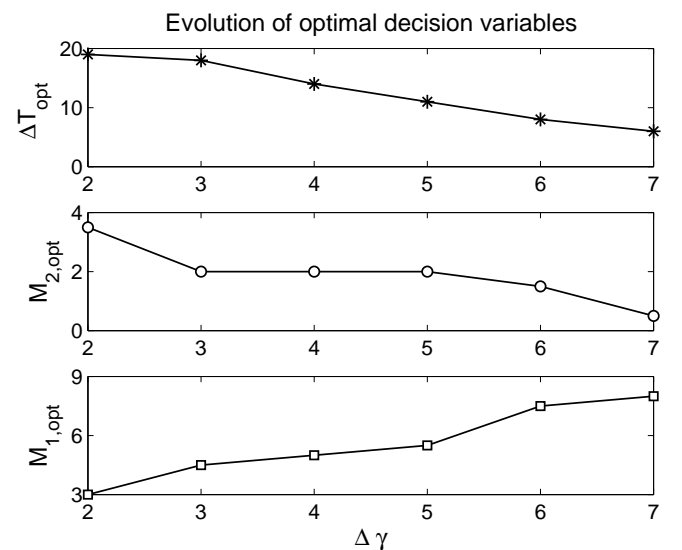

(b) $\left(\Delta T, M_{k}\right)$ strategy

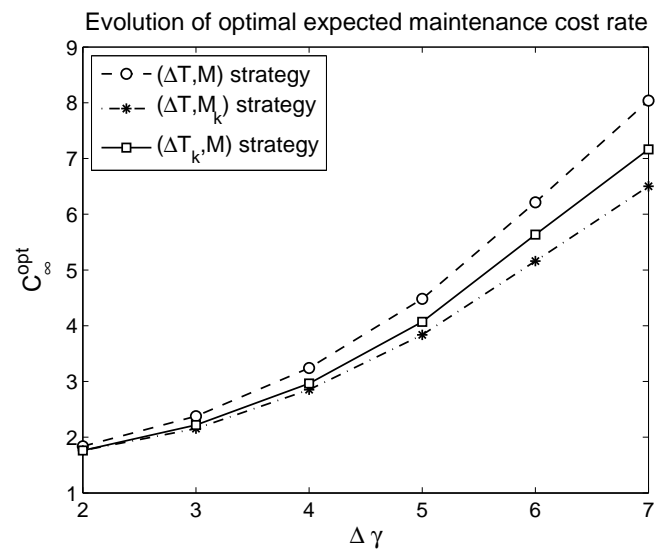

(c) Adaptive strategies vs. $(\Delta T, M)$ strategy

Figure 14: Sensitivity to the stress level of environment

\subsubsection{Sensitivity to the transition speed of environmental states}

For this case study, the stress level of the states $e_{t_{i}}=1$ et $e_{t_{i}}=2$ are fixed at $\gamma_{1}=0$ and $\gamma_{2}=6$ respectively. We vary the transition speed of environmental state (i.e., $\alpha=0.003,0.03,0.06,0.09,0.12$ ) and we investigate the corresponding evolutions of the optimal decision variables and the optimal expected maintenance cost rate of the considered strategies. Recall that the higher the value of $\alpha$, the more the transition of environment is quick. The results of this case study are shown in Figure 15.

We can see in Sub-figure 15c that the adaptive maintenance strategies can lead to substantial saving in maintenance cost compared to the $(\Delta T, M)$ strategy when the transition speed is low. But their 

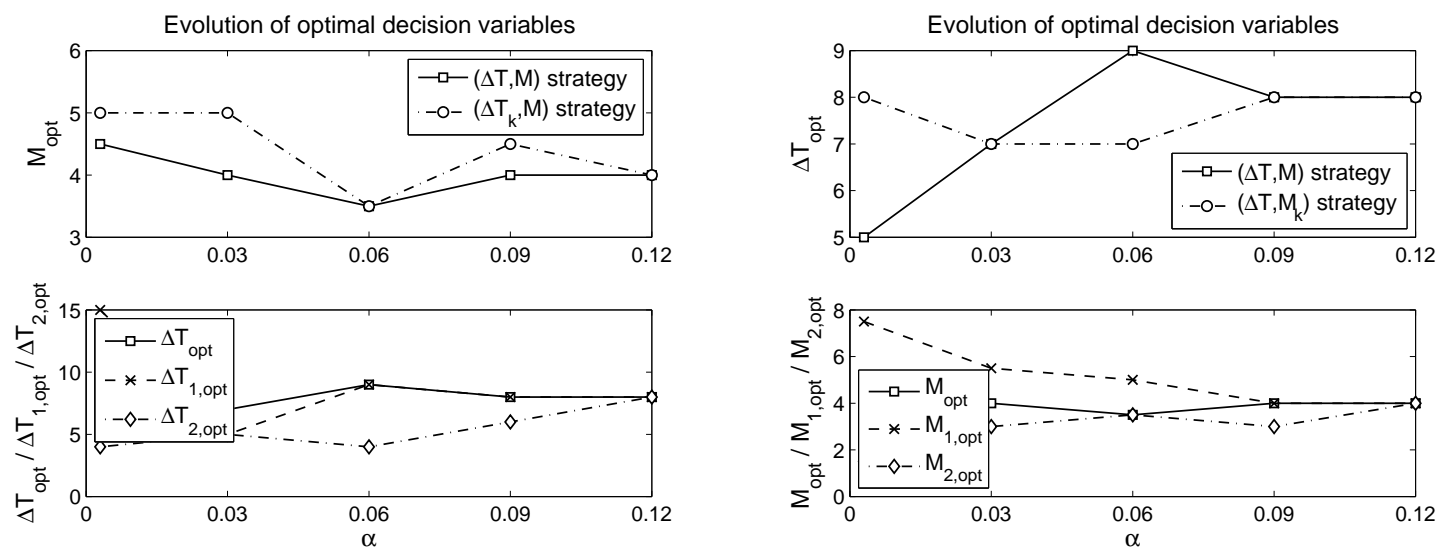

(a) $\left(\Delta T_{k}, M\right)$ strategy

(b) $\left(\Delta T, M_{k}\right)$ strategy

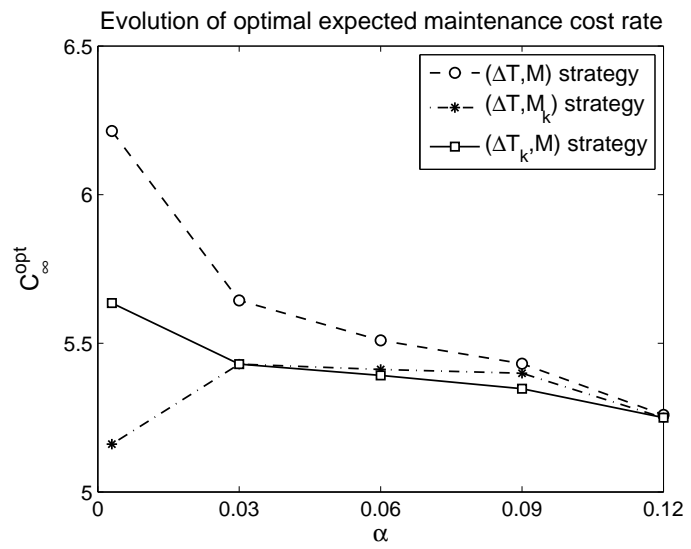

(c) Adaptive strategies vs. $(\Delta T, M)$ strategy

Figure 15: Sensitivity to the transition speed of environmental states

profit decreases, and eventually the optimal cost rates of the adaptive strategies converge exactly to the one of the $(\Delta T, M)$ strategy (see also Sub-figures $15 \mathrm{a}$ and $15 \mathrm{~b}$ for the evolution of the optimal decision variables). This results is comprehensible when we observe the system behavior illustrated as in Figure 1. When the environmental state varies slowly (see Sub-figure 1a), the adaptive strategies can hold pace with the change of environment, and hence their current conditions are really taken into account in maintenance decision making. Since the current states of environment obviously contain significant information about the system health, a maintenance decision adaptive to these current states can bring out more profit in cost saving. On the contrary, when the states of environment change too 
quickly, e.g., many times in a cycle lifetime of system (see figure 1b), the adaptive maintenance decision cannot (even impossible) be in tune with the variation of current environmental condition. As such, instead of relying on the current environmental state, these policies have to use its average behavior in maintenance decisions. In other words, these adaptive strategies turn exactly to the $(\Delta T, M)$ strategy, and their optimal cost rates become identical. In fact, the environment state that changes many times in a cycle system lifetime can make the degradation behavior of system more stable (i.e., lower variance) than when it change more slowly (see. Sub-figures 1c and 1d). That's why the optimal cost rate of $(\Delta T, M)$ strategy decreases with respect to the transition speed (see Sub-figure 15c). And this is also the reason for which the $\left(\Delta T, M_{k}\right)$ strategy is more efficient than the $\left(\Delta T_{k}, M\right)$ strategy when the transition speed of environment state is low enough and becomes less advantageous when this speed increases.

\subsection{Sensitivity to the estimation errors made on parameters of degradation process}

The sensitivity studies in previous sections are based on the assumption of perfect estimation of the model parameters. However, in many practical applications, the data collection can be quite difficult due to the lack of data and the measurement variability. This can lead to the errors in parameters estimation, and hence a loss in the performance of maintenance strategies. This section therefore aims to analyze the impact of the estimation errors made on parameters of degradation process on the maintenance cost of the considered strategies. This study shall help us to show the robustness of the adaptive maintenance strategies when the estimations of the system characteristics are biased.

The same data set in Section 4 is reused: $C=0.015, n=0.35, b_{b}=3.9, \sigma_{w}^{2}=2.53, \Delta t=0.2$, $\gamma_{1}=0, \gamma_{2}=6, \alpha=0.003, \beta_{0}=0.06, \beta_{1}=1.25, \sigma_{v}=0.4, N_{s}=3000, N_{h}=4000, C_{i}=8, C_{p}=50$, $C_{c}=100$ and $C_{d}=25$. We set each parameter of the degradation process (i.e., $C, n, b_{b}$ or $\sigma_{w}$ ) to its value corresponding to a quite important relative error of $-10 \%$ or $+10 \%$ whilst keeping the other parameters fixed, and we consider the cost rate of each adaptive maintenance strategies. Tables 1 and 2 represent the impact of the estimation errors on the maintenance cost of the $\left(\Delta T_{k}, M\right)$ strategy and the $\left(\Delta T, M_{k}\right)$ strategy respectively. The cost rate with uncertainties in each table is the one calculated at the optimal decision variables corresponding to the case of unbiased estimation (i.e., estimation error $=0 \%$ ). We can see clearly that the estimation error reduces the performance of the maintenance 
Table 1: Impact of the parameter estimation errors on the maintenance cost of $\left(\Delta T_{k}, M\right)$ strategy

\begin{tabular}{|c|c|c|c|c|c|c|c|c|}
\hline Parameter & \multicolumn{2}{|c|}{$C$} & \multicolumn{2}{|c|}{$n$} & \multicolumn{2}{|c|}{$b_{b}$} & \multicolumn{2}{c|}{$\sigma_{w}$} \\
\hline \hline Estimation error & $-10 \%$ & $+10 \%$ & $-10 \%$ & $+10 \%$ & $-10 \%$ & $+10 \%$ & $-10 \%$ & $+10 \%$ \\
$C_{\infty}^{\Delta T_{k}, M}\left(\Delta T_{1, \text { opt }}, \Delta T_{2, \text { opt }}, M_{\text {opt }}\right)$ & 4.9397 & 5.8969 & 4.3341 & 6.7294 & 5.3063 & 5.6607 & 4.2442 & 6.7934 \\
$C_{\infty}^{\Delta T_{k}, M}$ with uncertainties & 5.0467 & 5.9606 & 4.4088 & 6.8270 & 5.3621 & 5.6829 & 4.3530 & 6.8287 \\
Relative loss & $2.12 \%$ & $1.07 \%$ & $1.69 \%$ & $1.43 \%$ & $1.04 \%$ & $0.39 \%$ & $2.50 \%$ & $0.52 \%$ \\
\hline
\end{tabular}

Table 2: Impact of the parameter estimation errors on the maintenance cost of $\left(\Delta T, M_{k}\right)$ strategy

\begin{tabular}{|c|c|c|c|c|c|c|c|c|}
\hline Parameter & \multicolumn{2}{|c|}{$C$} & \multicolumn{2}{c|}{$n$} & \multicolumn{2}{|c|}{$b_{b}$} & \multicolumn{2}{c|}{$\sigma_{w}$} \\
\hline \hline Estimation error & $-10 \%$ & $+10 \%$ & $-10 \%$ & $+10 \%$ & $-10 \%$ & $+10 \%$ & $-10 \%$ & $+10 \%$ \\
$C_{\infty}^{\Delta T, M_{k}}\left(\Delta T_{\text {opt }}, M_{1, \text { opt }}, M_{2, \text { opt }}\right)$ & 4.5096 & 5.4242 & 4.0177 & 6.1098 & 4.8337 & 5.2011 & 3.9957 & 6.2204 \\
$C_{\infty}^{\Delta T, M_{k} \text { with uncertainties }}$ & 4.5719 & 5.4309 & 4.2195 & 6.3789 & 4.8457 & 5.2035 & 4.1087 & 6.2583 \\
Relative loss & $1.36 \%$ & $0.12 \%$ & $4.78 \%$ & $4.22 \%$ & $0.25 \%$ & $0.05 \%$ & $2.76 \%$ & $0.61 \%$ \\
\hline
\end{tabular}

strategies, however the loss is relatively small (less than $2.5 \%$ and $4.78 \%$ of relative loss for the $\left(\Delta T_{k}, M\right.$ ) strategy and the $\left(\Delta T, M_{k}\right)$ strategy respectively in the worst case). The adaptive strategies seem to be robust to uncertainties made on the parameters estimation of the system degradation process. However, to have a more definitive conclusion on the robustness of these strategies, we compare their cost rate with uncertainties with the optimal cost rate of $(\Delta T, M)$ strategy as in Table 3 .

Table 3: Robustness of the adaptive maintenance strategies

\begin{tabular}{|c|c|c|c|c|c|c|c|c|}
\hline Parameter & \multicolumn{2}{|c|}{$C$} & \multicolumn{2}{c|}{$n$} & \multicolumn{2}{c|}{$b_{b}$} & \multicolumn{2}{c|}{$\sigma_{w}$} \\
\hline \hline Estimation error & $-10 \%$ & $+10 \%$ & $-10 \%$ & $+10 \%$ & $-10 \%$ & $+10 \%$ & $-10 \%$ & $+10 \%$ \\
$C_{\infty}^{\Delta T, M_{k}}$ with uncertainties & 4.5719 & 5.4309 & 4.2195 & 6.3789 & 4.8457 & 5.2035 & 4.1087 & 6.2583 \\
$C_{\infty}^{\Delta T_{k}, M}$ with uncertainties & 5.0467 & 5.9606 & 4.4088 & 6.8270 & 5.3621 & 5.6829 & 4.3530 & 6.8287 \\
$C_{\infty}^{\Delta T, M}\left(\Delta T_{\text {opt }}, M_{\text {opt }}\right)$ & 5.5088 & 6.5620 & 4.7795 & 7.4595 & 5.8515 & 6.1820 & 4.6002 & 7.4797 \\
\hline
\end{tabular}

The result shows that with a relative error of $+10 \%$ or $-10 \%$ in parameters estimation, the $\left(\Delta T_{k}, M\right)$ strategy and the $\left(\Delta T, M_{k}\right)$ strategy always assure a good advantage compared to the $(\Delta T, M)$ strategy. This affirms the robustness of the proposed adaptive maintenance strategies.

\subsection{General discussions on the performance of the adaptive maintenance strategies}

From the above numerical results as well as the structures of the considered maintenance strategies, we can give some conclusions on their performance and their applicable condition as follows. 
Obviously, a maintenance strategy with multi-periods of inspection and/or with multi-thresholds for preventive replacement is more flexible, more general and hence more profitable in most situations when compared with the classical periodic inspection and replacement strategy. This gain comes from the abilities to adapt to the current variation of operating environment. In fact, the environment characteristics, as well as the maintenance costs, have significant impact on the effectiveness of an adaptive maintenance decision. A time-based adaptation approach is adequate for a system that operates under an environment with quite high speed of state transition and that requires a high cost to monitor and preventively replace. While a condition-based adaptation approach is particularly effective when the operating environment is more stressful, has low transition speed between the states, when the cost of inspection and preventive replacement are not expensive, as well as when the cost rate of system inactivity is higher. The adaptive strategies are also robust for not too high uncertainties made on the estimation of the system characteristics. Notwithstanding the above, when the characteristic of environment are unknown, the choice of an adaptation approach has to be cautious and should rely on an analysis of maintenance cost saving to ensure the best profit.

\section{Conclusions}

The present paper builds a general degradation and measurement model that describes many realistic aspects of single-unit systems operating under variable environment and indirect condition monitoring. The proposed model reflects the physical nature of degradation phenomenon, the relation among the true degradation and diagnostic and environmental covariates, as well as direct and indirect nature of condition monitoring. The true degradation of system is hidden and is recovered in realtime from noisy diagnostic covariates thanks to a so-called particle filter technique. We introduce two maintenance strategies where the decision rule is based on the estimated degradation state and adapts to the current condition or operating environment. In the first strategy, the adaption approach to environmental states relies on the inspection period (i.e. time-based approach), while in the second, it relies on the preventive replacement threshold (i.e. condition-based approach). These strategies reflect an efficient exploitation of many different types of condition information in maintenance decisionmaking. Since the cost models of these strategies cannot be solved analytically, we propose some algorithms based on particle filter technique and Monte Carlo simulation to overcome the problem. 
The speed and the effectiveness of these algorithms show that the numerical method is an alternative and useful tool to treat complex maintenance problems. The sensitivity studies in the paper allows us to show the performance and the robustness of the proposed strategies, as well as to give a trend to adjust the strategies towards their optimal solution when the system characteristics and/or the operating environment change. Also, we have shown that the environment adaptive policies are more general and more flexible than the classical periodic inspection and replacement strategy, and hence that they always guarantee efficient maintenance cost savings. However, except for some extreme situations, there is no obvious and general way to choose between time-based and condition-based adaptation approaches, and thus the choice has to be cautious and should be decided through analyzing the maintenance cost saving.

The data used in this paper are objective information collected through condition monitoring. Besides these data, subjective information (i.e. expert knowledge, customer feedback, etc.) is also useful. Considering all types of information in maintenance decision-making is obviously a challenge task, but promises significant cost savings and opens perspective for further research.

[1] J.D. Kalbfleisch and R.L. Prentice, The statistical analysis of failure time data. Wiley, New York, 2002.

[2] W. Wang, "Condition-based maintenance modelling," in Complex System Maintenance Handbook, Springer Series in Reliability Engineering, pp. 111-131, Springer London, 2008.

[3] J.P. Kharoufeh, "Explicit results for wear processes in a markovian environment," Operations Research Letters, vol. 31, no. 3, pp. 237-244, 2003.

[4] E. Deloux, B. Castanier, and C. Bérenguer, "Predictive maintenance policy for a gradually deteriorating system subject to stress," Reliability Engineering \& System Safety, vol. 94, no. 2, pp. 418-431, 2009.

[5] X. Zhao, M. Fouladirad, C. Bérenguer, and L. Bordes, "Condition-based inspection/replacement policies for non-monotone deteriorating systems with environmental covariates," Reliability Engineering 8 System Safety, vol. 95, no. 8, pp. 921-934, 2010.

[6] K.T. Huynh, A. Barros, C. Bérenguer, and I.T. Castro, "A periodic inspection and replacement policy for systems subject to competing failure modes due to degradation and traumatic events," 
Reliability Engineering \&3 System Safety, vol. 96, no. 4, pp. 497-508, 2011.

[7] W. Wang, "A prognosis model for wear prediction based on oil-based monitoring," Journal of the Operational Research Society, vol. 58, no. 7, pp. 887-893, 2006.

[8] Z. Xu, Y. Ji, and D. Zhou, "Real-time reliability prediction for a dynamic system based on the hidden degradation process identification," IEEE Transactions on Reliability, vol. 57, no. 2, pp. 230-242, 2008.

[9] F. Cadini, E. Zio, and D. Avram, "Model-based monte carlo state estimation for condition-based component replacement," Reliability Engineering 83 System Safety, vol. 94, no. 3, pp. 752-758, 2009.

[10] K.T. Huynh, A. Barros, and C. Bérenguer, "Maintenance decision-making for systems operating under indirect condition monitoring: value of online information and impact of measurement uncertainty," IEEE Transactions on Reliability, vol. 61, no. 2, pp. 410-425, 2012.

[11] E. Myötyri, U. Pulkkinen, and K. Simola, "Application of stochastic filtering for lifetime prediction," Reliability Engineering \&3 System Safety, vol. 91, no. 2, pp. 200-208, 2006.

[12] E. Zio and G. Peloni, "Particle filtering prognostic estimation of the remaining useful life of nonlinear components," Reliability Engineering 83 System Safety, vol. 96, no. 3, pp. 403-409, 2011.

[13] Y. Yang and G.A. Klutke, "Improved inspection schemes for deteriorating equipment," Probability in the Engineering and Informational Sciences, vol. 14, no. 04, pp. 445-460, 2000.

[14] A. Grall, L. Dieulle, C. Bérenguer, and M. Roussignol, "Continuous-time predictive-maintenance scheduling for a deteriorating system," IEEE Transactions on Reliability, vol. 51, no. 2, pp. 141$150,2002$.

[15] C. Barker and M. Newby, "Optimal non-periodic inspection for a multivariate degradation model," Reliability Engineering ES System Safety, vol. 94, no. 1, pp. 33-43, 2009.

[16] A. Ponchet, M. Fouladirad, and A. Grall, "Assessment of a maintenance model for a multideteriorating mode system," Reliability Engineering \&3 System Safety, vol. 95, no. 11, pp. 1244$1254,2010$.

[17] E. Deloux, B. Castanier, and C. Bérenguer, "Environmental information adaptive condition-based maintenance policies," Structure and Infrastructure Engineering, vol. 8, no. 4, pp. 373-382, 2012. 
[18] X. Wang, "Physical degradation models," in Encyclopedia of Statistics in Quality and Reliability (Eds F. Ruggeri, R. Kenett and F. W. Faltin), pp. 1356-1361, 2007.

[19] F. Kozin and J.L. Bogdanoff, "Probabilistic models of fatigue crack growth: results and speculations," Nuclear Engineering and Design, vol. 115, no. 1, pp. 143-171, 1989.

[20] M. Bigerelle and A. Iost, "Bootstrap analysis of FCGR, application to the Paris relationship and to lifetime prediction," International Journal of Fatigue, vol. 21, no. 4, pp. 299-307, 1999.

[21] I.C. Whittaker and S.C. Saunders, "Application of Reliability Analysis to Aircraft Structures Subject to Fatigue Crack Growth and Periodic Structural Inspection," Tech. Rep. AFML-TR-7392, Air Force Material Laboratories, 1973.

[22] Y.K. Lin and J.N. Yang, "On statistical moments of fatigue crack propagation," Engineering Fracture Mechanics, vol. 18, no. 2, pp. 243-256, 1983.

[23] F. Kozin and J.L. Bogdanoff, "A critical analysis of some probabilistic models of fatigue crack growth," Engineering Fracture Mechanics, vol. 14, no. 1, pp. 59-89, 1981.

[24] J.N. Yang and S.D. Manning, "A simple second order approximation for stochastic crack growth analysis," Engineering Fracture Mechanics, vol. 53, no. 5, pp. 677-686, 1996.

[25] J. Xing, O.P. Zhong, and Y.J. Hong, "A simple log normal random process approach of the fatigue crack growth considering the distribution of initial crack size and loading condition," International Journal of Pressure Vessels and Piping, vol. 74, no. 1, pp. 7-12, 1997.

[26] W. Wu and C. Ni, "A study of stochastic fatigue crack growth modeling through experimental data," Probabilistic Engineering Mechanics, vol. 18, no. 2, pp. 107-118, 2003.

[27] W.F. Wu and C.C. Ni, "Probabilistic models of fatigue crack propagation and their experimental verification," Probabilistic Engineering Mechanics, vol. 19, no. 3, pp. 247-257, 2004.

[28] S. Özekici, "Optimal maintenance policies in random environments," European Journal of Operational Research, vol. 82, no. 2, pp. 283-294, 1995.

[29] D.R. Cox, "Regression models and life-tables," Journal of the Royal Statistical Society. Series B (Methodological), vol. 34, no. 2, pp. 187-220, 1972.

[30] K. Simola and U. Pulkkinen, "Models for non-destructive inspection data," Reliability Engineering and System Safety, vol. 60, no. 1, pp. 1-12, 1998.

[31] J.M. van Noortwijk and J.A.M. van der Weide, "Applications to continuous-time processes of 
computational techniques for discrete-time renewal processes," Reliability Engineering 83 System Safety, vol. 93, no. 12, pp. 1853-1860, 2008.

[32] H.M. Wagner, Principles of Operations Research, Second Edition. Prentice-Hall, Englewood Cliffs, N.J, 1975.

[33] H.C. Tijms, A first course in stochastic models. Wiley, New York, 2003.

[34] L. Mann, A. Saxena, and G.M. Knapp, "Statistical-based or condition-based preventive maintenance?," Journal of Quality in Maintenance Engineering, vol. 1, no. 1, pp. 46-59, 1995.

[35] M.E. Orchard and G.J. Vachtsevanos, "A particle-filtering approach for on-line fault diagnosis and failure prognosis," Transactions of the Institute of Measurement \& Control, vol. 31, no. 3-4, p. 221,2009 .

[36] Y. Sun, L. Ma, J. Mathew, W. Wang, and S. Zhang, "Mechanical systems hazard estimation using condition monitoring," Mechanical Systems and Signal Processing, vol. 20, no. 5, pp. 1189-1201, 2006 .

[37] P. Baruah and R.B. Chinnam, "HMMs for diagnostics and prognostics in machining processes," International Journal of Production Research, vol. 43, no. 6, pp. 1275-1293, 2005.

[38] X.S. Si, W. Wang, C.H. Hu, and D.H. Zhou, "Remaining useful life estimation - a review on the statistical data driven approaches," European Journal of Operational Research, vol. 213, no. 1, pp. 1-14, 2011.

[39] M.S. Arulampalam, S. Maskell, N. Gordon, and T. Clapp, "A tutorial on particle filters for online nonlinear/non-gaussian bayesian tracking," IEEE Transactions on Signal Processing, vol. 50, no. 2, pp. 174-188, 2002.

[40] A. Doucet, N. De Freitas, and N. Gordon, Sequential Monte Carlo methods in practice. Springer Verlag, 2001.

[41] G. Kitagawa, "Monte carlo filter and smoother for non-gaussian nonlinear state space models," Journal of computational and graphical statistics, vol. 5, no. 1, pp. 1-25, 1996.

[42] A.D. Belegundu and T.R. Chandrupatla, Optimization concepts and applications in engineering. Cambridge University Press, 2011. 